\title{
Two Parallel Olfactory Pathways for Processing General Odors in a Cockroach
}

\author{
Hidehiro Watanabe ${ }^{1 *}$, Hiroshi Nishino ${ }^{2}$, Makoto Mizunami ${ }^{3}$ and Fumio Yokohari ${ }^{1}$ \\ ${ }^{1}$ Division of Biology, Department of Earth System Science, Fukuoka University, Fukuoka, Japan, ${ }^{2}$ Research Institute for \\ Electronic Science, Hokkaido University, Sapporo, Japan, ${ }^{3}$ Faculty of Science, Hokkaido University, Sapporo, Japan
}

\section{OPEN ACCESS}

Edited by:

Takao K. Hensch,

Harvard University, USA

Reviewed by:

Hokto Kazama,

RIKEN Brain Science Institute, Japan

Daniel W. Wesson,

University of Florida, USA

${ }^{*}$ Correspondence:

Hidehiro Watanabe nabehide@fukuoka-u.ac.jp

Received: 17 January 2017 Accepted: 18 April 2017 Published: 05 May 2017

Citation:

Watanabe $\mathrm{H}$, Nishino $\mathrm{H}$, Mizunami $\mathrm{M}$ and Yokohari F (2017) Two Parallel Olfactory Pathways for Processing General Odors in a Cockroach. Front. Neural Circuits 11:32. doi: 10.3389/fncir.2017.00032
In animals, sensory processing via parallel pathways, including the olfactory system, is a common design. However, the mechanisms that parallel pathways use to encode highly complex and dynamic odor signals remain unclear. In the current study, we examined the anatomical and physiological features of parallel olfactory pathways in an evolutionally basal insect, the cockroach Periplaneta americana. In this insect, the entire system for processing general odors, from olfactory sensory neurons to higher brain centers, is anatomically segregated into two parallel pathways. Two separate populations of secondary olfactory neurons, type1 and type2 projection neurons (PNs), with dendrites in distinct glomerular groups relay olfactory signals to segregated areas of higher brain centers. We conducted intracellular recordings, revealing olfactory properties and temporal patterns of both types of PNs. Generally, type1 PNs exhibit higher odorspecificities to nine tested odorants than type2 PNs. Cluster analyses revealed that odor-evoked responses were temporally complex and varied in type1 PNs, while type2 PNs exhibited phasic on-responses with either early or late latencies to an effective odor. The late responses are 30-40 ms later than the early responses. Simultaneous intracellular recordings from two different PNs revealed that a given odor activated both types of PNs with different temporal patterns, and latencies of early and late responses in type2 PNs might be precisely controlled. Our results suggest that the cockroach is equipped with two anatomically and physiologically segregated parallel olfactory pathways, which might employ different neural strategies to encode odor information.

Keywords: insect, olfaction, parallel processing, projection neurons, temporal pattern, intracellular recording, simultaneous intracellular recording, antennal lobe

\section{INTRODUCTION}

Information processing along parallel pathways is a common feature of animal sensory systems, enabling fast and reliable representations of highly complex and dynamic sensory information. Parallel sensory pathways typically extract and process different types of stimulus parameters. In the vertebrate olfactory system, general and pheromonal odors are processed in the main olfactory and vomeronasal pathways, respectively (Tirindelli et al., 2009). Within the main olfactory pathway, mitral and tufted cells in the olfactory bulb process odor qualities and timing information, respectively (Nagayama et al., 2004; Igarashi et al., 2012).

Because the olfactory bulb of vertebrates and the antennal lobe (AL) of insects are functionally similar neural substrates, the insect olfactory system consisting of a small number of identifiable 
neurons provides a useful model for studying the neural basis of parallel olfactory processing (Hildebrand and Shepherd, 1997; Knaden and Hansson, 2014). In insects, olfactory sensory neurons (OSNs) are housed in the antennal sensilla and project to glomeruli in the AL. In each glomerulus, a large number of OSNs, which express a cognate type of odorant receptor relay onto a moderate number of secondary olfactory interneurons, projection neurons (PNs), and local interneurons (LNs). PNs send olfactory information to higher brain centers, mushroom bodies (MBs) and lateral horns (LHs). Conversely, LNs in the AL interconnect glomeruli and contribute to odor-induced spatiotemporal activity patterns of PNs (Wilson et al., 2004; Watanabe et al., 2012a).

Two categories of parallel olfactory pathways have been proposed in insects (Galizia and Rössler, 2010): "segregated parallel pathways," which process different odors in different pathways, and "dual parallel pathways," which process different parameters of a given odor. For example, the pathway for processing species-specific odors, such as pheromones, is anatomically segregated from the pathway for processing general odors from antennal sensilla to higher brain centers (Burrows et al., 1982; Nishikawa et al., 2012; Nishino et al., 2012a). The dual parallel pathways have been observed in hymenopteran insects. In honeybees and ants, the axons of PNs innervating glomeruli in the dorsal AL hemilobe run through the lateral AL tract (1-ALT), whereas those of $\mathrm{PN}$ axons innervating glomeruli in the ventral AL hemilobe run through the medial ALT (m-ALT) (Kirschner et al., 2006; Zube et al., 2008). Because both l-ALT and $\mathrm{m}$-ALT PNs receive olfactory inputs in part from common sensilla (Kelber et al., 2006) and their projection fields partially overlap, their anatomical subdivisions are unclear. A given odor stimulus recruits both PN types, and studies have suggested that 1-ALT and m-ALT PNs might extract different parameters of a given olfactory stimulus (Muller et al., 2002; Krofczik et al., 2008; Galizia and Rössler, 2010; Rössler and Zube, 2011; Brill et al., 2013, 2015; Rössler and Brill, 2013). However, the mechanisms by which parallel pathways extract and encode different parameters of olfactory information remain unclear.

The current study sought to determine the neural mechanisms underlying parallel olfactory processing in insects. In the cockroach Periplaneta americana, the $\mathrm{AL}$ equips two sex pheromone-sensitive glomeruli and 203 ordinary glomeruli which process general odors. The ordinary glomeruli have been classified into two glomerular groups: the antero-dorsal and postero-ventral groups (Watanabe et al., 2010). Because the antero-dorsal and postero-ventral group glomeruli receive olfactory inputs from OSNs in different types of sensilla that exhibit different odor spectra, they are morpho-functionally segregated (Fujimura et al., 1991; Watanabe et al., 2012a,b). The current study revealed that olfactory signals processed in postero-ventral and anterodorsal group glomeruli are relayed by two morphologically distinct types of uniglomerular PNs, termed type1 and type2 PNs, respectively (Strausfeld and Li, 1999a; Watanabe et al., 2012a). We used two approaches that provided insight into the neural mechanisms underlying parallel olfactory processing: (1) we examined the anatomical and physiological features of PNs in each pathway and (2) we performed simultaneous intracellular recording from two different types of PNs.

\section{MATERIALS AND METHODS}

\section{Experimental Animals}

Male adult cockroaches $P$. americana were obtained from laboratory colonies maintained under a 12:12-h light:dark cycle at $28^{\circ} \mathrm{C}$ in Fukuoka University.

\section{Tracer Application to Projection Neurons and OSN Afferents}

After cockroaches were anesthetized on ice, the head capsule was incised and fixed to a wax plate anterior side up. The cuticle between the two antennae was squarely cut and the overlaying muscles and tracheae on the brain were removed. In the cockroach, axons of all uniglomerular PNs run through the m-ALT or nearby tracts (Malun et al., 1993; Strausfeld and Li, 1999a). For retrograde staining of PNs, a tapered glass electrode coated with moisture-absorbed crystals from microemerald (dextran fluorescein with biotin, 3000 MW, D-7156, ThermoFisher Scientific, Waltham, MA, USA) was manually inserted into the m-ALT. After application of the fluorescent dye, the opening of the head was covered with a previously cut square of cuticle. Antennal afferents then underwent anterograde staining to visualize glomeruli as previously described (Nishino et al., 2009; Watanabe et al., 2010). The antennal nerve on the ipsilateral side was exposed and excised at the flagellar base. The proximal cut end of the antennal nerve was inserted into a tapered glass capillary filled with a $10 \%$ aqueous solution of micro-ruby (dextran tetramethyl rhodamine with biotin, 3000 MW, D-7162, ThermoFisher Scientific). The specimen was incubated in a moist chamber under dark conditions at $4^{\circ} \mathrm{C}$ for 2 days. After incubation, the brain was dissected from the head capsule. The isolated brain was fixed in a $4 \%$ formaldehyde solution at $4^{\circ} \mathrm{C}$ for 3-5 h, dehydrated in an ascending ethanol series (from $70 \%$ to $100 \%$ ), then cleared in methyl salicylate.

\section{Single and Simultaneous Intracellular Recordings}

The method used for intracellular recording and staining from individual PNs of the cockroach was modified from methods reported in our previous studies (Nishino et al., 2003, 2011, 2012a; Watanabe et al., 2012a). Cockroaches were briefly anesthetized and mounted on experimental chambers with lowmelting point wax. Each antenna was immobilized by threading a plastic ring (diameter: $1 \mathrm{~mm}$ ). The cuticle between the two antennae was opened, and the brain was exposed. After the brain sheath had been softened with Actinase E (Kaken Seiyaku, Tokyo, Japan), the brain and platinum ground electrode were immersed in a cockroach saline solution $(\mathrm{NaCl} 210.2 \mathrm{mM}, \mathrm{KCl} 3.1 \mathrm{mM}$, $\mathrm{CaCl}_{2} 1.8 \mathrm{mM}, \mathrm{NaH}_{2} \mathrm{PO}_{4} 0.2 \mathrm{mM}, \mathrm{Na}_{2} \mathrm{HPO}_{4} 1.8 \mathrm{mM}$, pH 7.2). To stabilize the brain, a glass rod was inserted into the cavity formed by removal of the esophagus. 
A borosilicate glass microelectrode pulled by a laser puller (P-2000; Sutter Instruments, Novato, CA, USA) was filled with $8 \%$ Lucifer Yellow (Sigma, St. Louis, MO, USA) or $10 \mathrm{mM}$ Alexa 647 (ThermoFisher Scientific) in $1 \mathrm{M} \mathrm{LiCl}$ (aqueous). An electrode was inserted into the cluster of PN somata located in the dorsal region of the AL (Figures 1A,B; Distler and Boeckh, 1997a,b; Watanabe et al., 2012a). In simultaneous intracellular recordings from two different PNs, two electrodes were filled with different fluorescent dyes (Lucifer Yellow and Alexa 647) and separately inserted into the ipsilateral AL. The neural activity of individual neurons was amplified (MEZ8301, Nihon Kohden, Tokyo, Japan) and displayed on an oscilloscope. Spikes were digitized by a PowerLab data acquisition system (AD Instruments Japan Inc., Nagoya, Japan).

\section{Olfactory Stimulation}

To attain PN response data efficiently, we carefully chose nine different odors that collectively cover the broad spectra of sensory neuronal responses. In the cockroach $P$. americana, most OSNs responding to general odors have been classified into eight groups based on similarities in the response spectra (Fujimura et al., 1991). In the current study, nine mono-molecule odorants, each eliciting strong excitatory effects in one of the eight OSN groups, were selected: pentanol, hexanol, octanol, nonanol, phenyl acetate, cineol, santalol, terpineol, and heptanoic acid. Pure solutions of each odorant were diluted 10 times with paraffin oil, and a small piece of filter paper was soaked with $40 \mu \mathrm{L}$ of one of the solutions and inserted into each nozzle.

Fresh air taken from outdoors via a diaphragm pump was cleaned with a cotton and charcoal filter and divided into two tubes; one passed through three bottles filled with distilled water (humidified air) and the other passed through three bottles filled with silica-gel (dried air). We used air at 50\% relative humidity by mixing the humidified and dried air. The flow-rate of air within the tube was maintained at $1 \mathrm{~L} / \mathrm{min}$ using a flowmeter. The tube was further divided into nine connected to nine glass nozzles containing nine different odorants. Odorant nozzles and a blank (control) nozzle were arbitrarily selected for stimulation by manual valve operation. The nozzle tip ( $5 \mathrm{~mm}$ diameter) was positioned approximately $10 \mathrm{~mm}$ from approximately the 30th antennal flagellomere, and the air around the preparation was continuously exhausted by a duct located in front of the antenna tip. The three-way solenoid valve was controlled by a stimulator (SEN7203, Nihon Kohden, Tokyo, Japan). We defined the timing of the solenoid valve opening by a stimulator as the odor onset. The stimulus period was set at $1 \mathrm{~s}$. We designed experiment to minimalize the number of olfactory stimulations to avoid the deterioration of recorded $\mathrm{PN}$ : each odorant was presented twice with a 30-s interval. After stimulation with a given odorant, a new nozzle containing another odorant was moved to the same position as the previous nozzle.

\section{Confocal Observations and Three-Dimensional Reconstruction}

After recording olfactory responses, the neuron was filled with fluorescent dye by injecting a hyperpolarizing current. After intracellular staining, the electrode was removed from the brain, and the head capsule was fixed on a wax plate. Antennal afferents then underwent anterograde staining using micro-ruby, as previously described. Contact between the specimen and the dye was maintained in a humid chamber at $4^{\circ} \mathrm{C}$ overnight. Subsequently, the double- or triple-stained brain was dissected from the head capsule. The isolated brain was fixed, dehydrated and cleared, as previously described.

The cleared specimens were examined with a confocal laser scanning microscope (LSM-510; Carl Zeiss, Jena, Germany) equipped with Argon and Helium-Neon lasers. Single neurons labeled by intracellular injection of Lucifer Yellow and/or Alexa 645 were, respectively, visualized using an Argon laser with a band-pass emission filter (505-550 nm) and/or a HeliumNeon laser with a long-pass emission filter (>650 nm). In addition, sensory afferents labeled with micro-ruby were acquired using a Helium-Neon laser with a band-pass emission filter (560-615 nm). Images were obtained using three different objectives: a Plan-Apochromat $10 \times / 0.7$ and $20 \times / 0.8$ objectives for low magnification images, and an oil-immersion PlanNeofluar $40 \times / 1.3$ were used for capturing high magnification images. All images were captured as $1024 \times 1024$ pixels. A series of TIFF-formatted optical sections were processed using image processing software (Amira 6.0, TGC, Berlin, Germany). Labeled neurons were traced with an image segmentation tool in Amira. The contrast and brightness of all images were adjusted appropriately using Adobe Photoshop CS3 and Illustrator CS3.

\section{Terminology}

In the male cockroach AL, all 203 ordinary glomeruli and two macroglomeruli can be unambiguously identified by the innervation patterns of $\mathrm{T} 1$ to $\mathrm{T} 10$ sensory tracts (Watanabe et al., 2010). The nomenclatures and detailed morphological features of glomeruli and glomerular groups were described in our previous studies (Watanabe et al., 2010, 2012b). Specifically, ordinary glomeruli belonging to the T1-T4 groups were morphologically, functionally, and developmentally segregated from those belonging to the T5-T10 groups (Salecker and Boeckh, 1995; Watanabe et al., 2010, 2012b). We termed the former and latter groups the 'antero-dorsal' and 'postero-ventral glomerular groups', respectively. The PN types were named according to terminology used in previous anatomical studies (Malun et al., 1993; Strausfeld and Li, 1999a; Nishino et al., 2012a). Each PN was named based on the innervating glomerulus. The medial and lateral calyces of the MB were defined as input sites of Kenyon cells (KCs), and each calyx was divided into four zones (I-III, IIIA) from the periphery to the base (Mizunami et al., 1998; Strausfeld and Li, 1999a,b). Brain orientation is shown with reference to the body axis. Nomenclatures and abbreviations of brain structures followed those established in the systematic nomenclature for the insect brain (Ito et al., 2014).

\section{Data Analyses}

To quantify spike numbers during a given time period, we used functions attached in Spike 2 ver.8.08 (CED, Cambridge, England). Odor responses were measured as an increase in spike frequency from the spontaneous level; $\mathrm{R}-\mathrm{R}_{0}$, where $\mathrm{R}$ or $\mathrm{R}_{0}$ was 

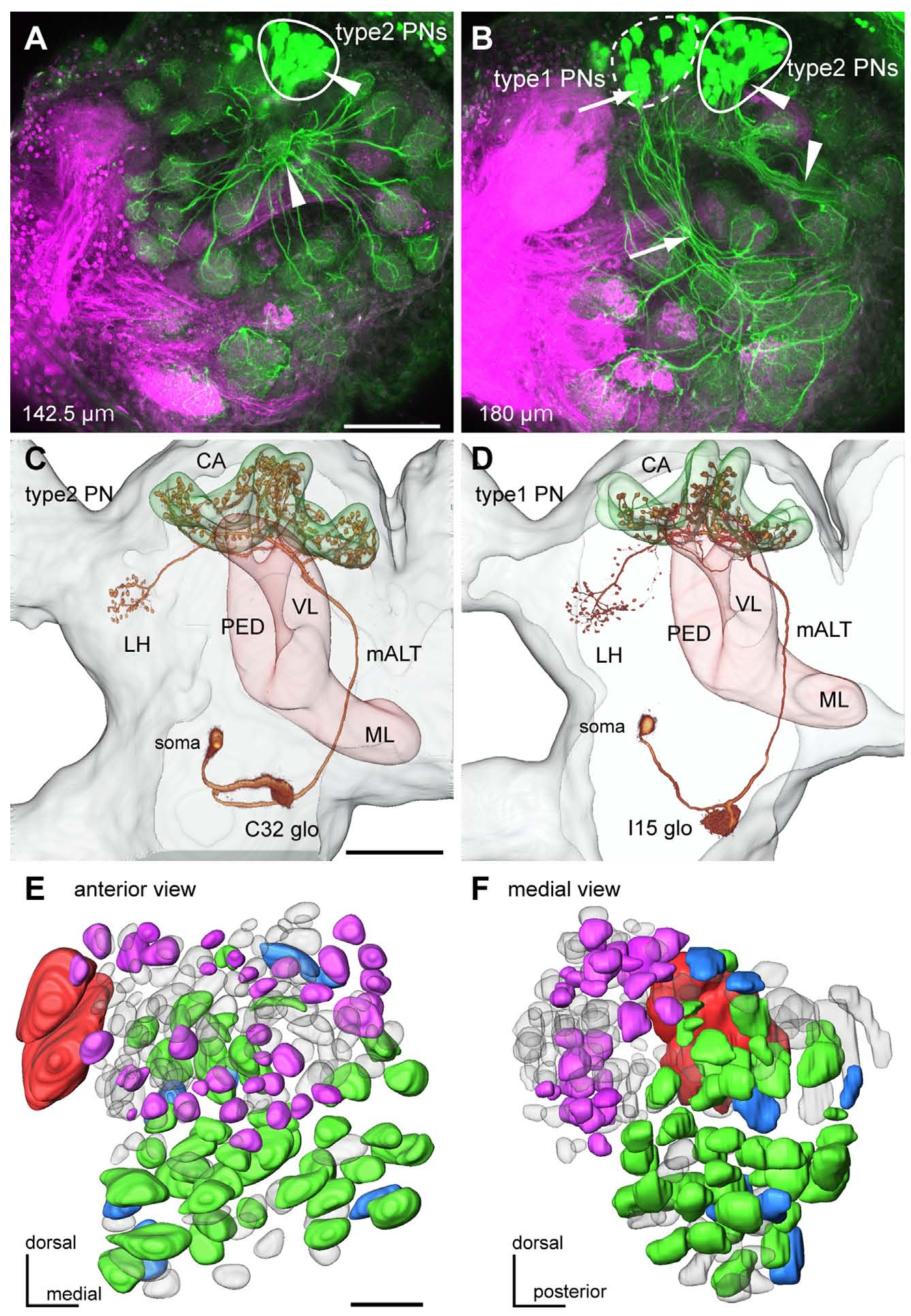

F medial view

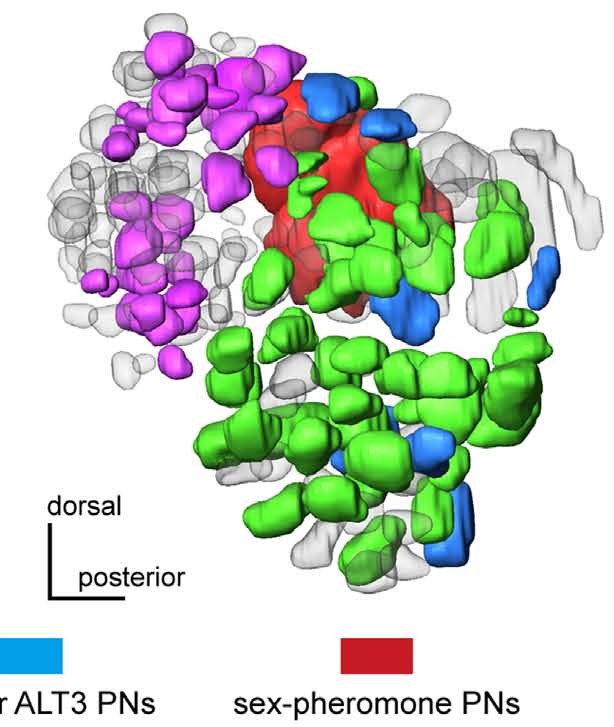

FIGURE 1 | Two major types of uniglomerular projection neurons (PNs) in the cockroach brain. (A,B) Mass staining of PNs in the antennal lobe (AL). PNs (green) were stained by dye injection into the medial ALT ( $m$-ALT) and olfactory sensory neurons (OSNs) (magenta) were labeled by anterograde staining from the antennal nerves. Axons of PNs form two different bundles in the AL (arrowheads and arrows in $\mathbf{A}, \mathbf{B}$ ), and their cell bodies separately cluster in the dorsal region of the AL. Loci of clusters of cell bodies correspond to the two major types of PNs; type1 and type2 PNs. Depth of serial optical image from anterior surface is

(Continued) 


\section{FIGURE 1 | Continued}

indicated in each panel. (C,D) 3D images of two major types of uniglomerular PNs. Axons of both type2 PN (C) and type1 PN (D) run through the m-ALT, anteriorly to the mushroom bodies (MB) peduncle (PED) and terminate in both MB calyces (CA) and lateral horn (LH). (E,F) PN type-specific glomerular organization, viewed anteriorly (E) and medially (F). Glomeruli innervated by intracellularly stained PNs are colored according to PN types. Type2 PNs specifically arborize in the antero-dorsal group glomeruli (magenta in E,F), whereas type1 PNs arborize in the postero-ventral group glomeruli (green in E,F). Glomeruli innervated by ALT2 and ALT3 uniglomerular PNs, which have been identified by Malun et al. (1993), are colored blue. Two macroglomeruli innervated by sex pheromone-sensitive PNs are colored red. Bars in $(\mathbf{A}, \mathbf{E})=100 \mu \mathrm{m}$, bar in $\mathbf{( C )}=200 \mu \mathrm{m}$
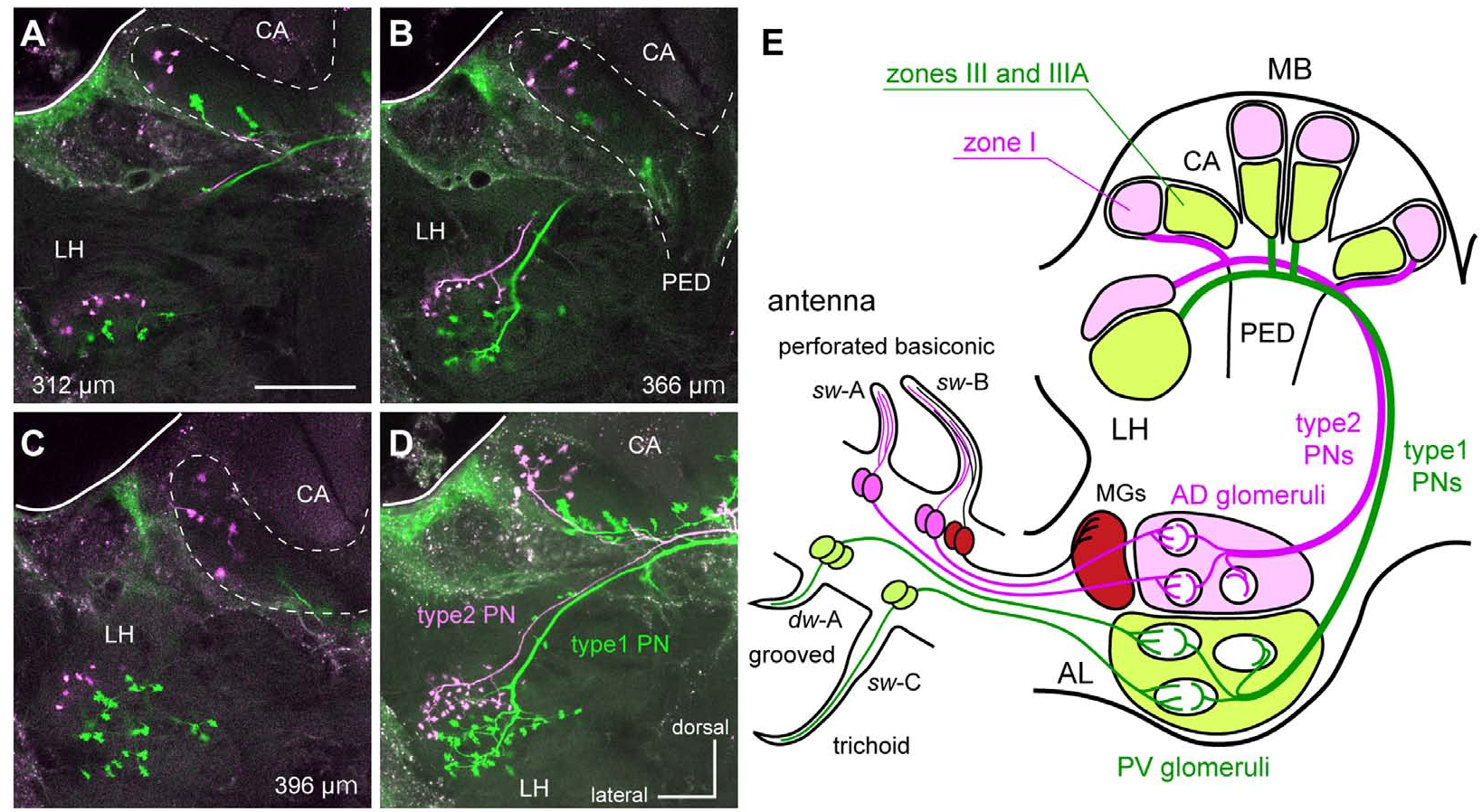

FIGURE 2 | The segregated parallel pathways from the periphery to higher brain centers in the cockroach brain. (A-D) A type1 PN (green) and a type2 PN (magenta) differentially labeled in the protocerebrum. Serial optical sections (A-C) and a stacked image (D) reveal that terminal regions of type1 PN are segregated from those of the type2 PN in the MB calyces (CA) and the LH. Depths of the serial optical images from anterior to posterior are indicated in each of panels (A-C). Twenty-five serial optical images obtained every 4- $\mu \mathrm{m}$ are stacked in (D). Bar in (A) $=100 \mu \mathrm{m}$. (E) Schematic drawing of parallel pathways from the periphery to higher brain center in the cockroach brain. OSNs in perforated basiconic sensilla (single-walled A [sw-A] and sw-B) selectively terminate in antero-dorsal group glomeruli (AD glomeruli), whereas those in trichoid sensilla (sw-C) and grooved basiconic sensilla (double-walled A [dw-A]) projects to the postero-ventral group glomeruli (PV glomeruli: Watanabe et al., 2012b). Therefore, type1 PNs with dendrites in PV glomeruli and type2 PNs with dendrites in AD glomeruli form two segregated parallel pathways from the periphery to higher brain centers. Nomenclatures of MB calyces were described in previous articles (Mizunami et al., 1998; Strausfeld and Li, 1999a,b). MGs, macroglomeruli; PED, pedunculus.

the number of spikes during the 1-s odor stimulation or during the 1-s pre-stimulation period, respectively. In each PN, odor intensity to a given odor was represented as the average $\mathrm{R}-\mathrm{R}_{0}$ of two trials. To identify odor response properties of recorded PNs, we performed hierarchical cluster analysis using the free programming software R v.3.3.2 (R Foundation for Statistical Computing, Vienna, Austria). Based on " $\mathrm{R}-\mathrm{R}_{0}$ " to nine tested odors, recorded PNs were classified into several groups using the cluster dendrogram expressed by Ward's method. Odor responses were regarded as excitatory if the $\mathrm{R}$ was more than two times higher than $\mathrm{R}_{0}$ in the two trials. In each recording, we quantified the number of effective odors that elicited excitatory responses to the PN. The recruitment rates of PNs were shown as a percentage of odor-activated PNs per odor. The percentages were separately calculated in type1 and type2 PNs. The number of effective odors and recruitment rates were statistically compared using the chi-square test or paired $t$-test.

Temporal activity patterns elicited by a given odor stimulus were summarized as raster plots and accumulated histograms with a bin of $10 \mathrm{~ms}$. After normalizing the histograms by dividing the number of spikes of each bin by the number of samples, we statistically compared histograms using the KolmogorovSmirnov test (KS-test). To compare differences of odor-induced temporal activity patterns across PN types and across odors, we performed the hierarchical cluster analysis (Ward's method) and the principal component analysis (PCA) using the "cluster" package in $\mathrm{R}$ software. In these analyses, we used excitatory responses to four different odorants (hexanol, octanol, phenyl acetate, and cineol) obtained by single intracellular recordings. In each response, the spike array during the 1-s odor stimulation 
A

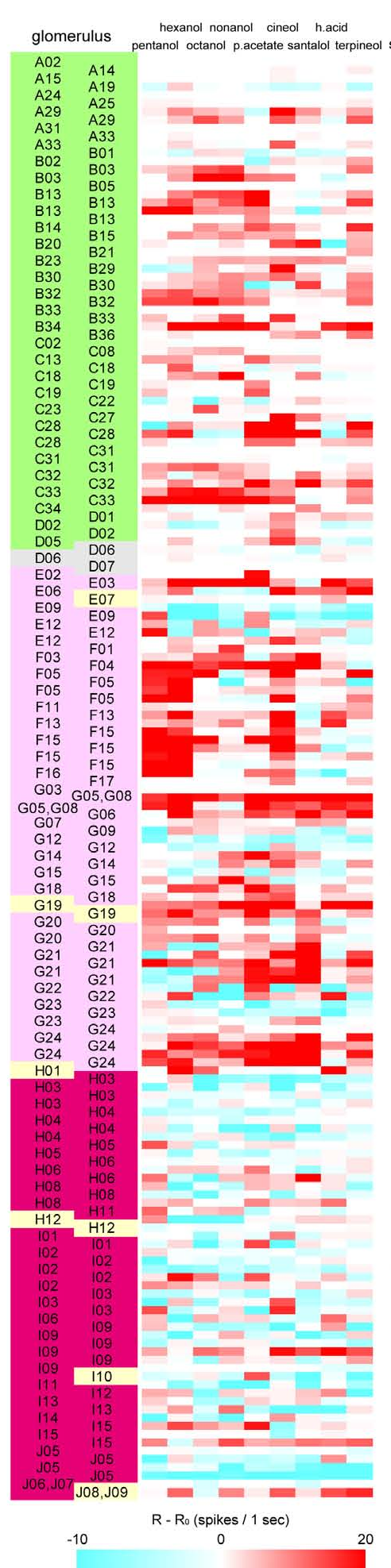

B

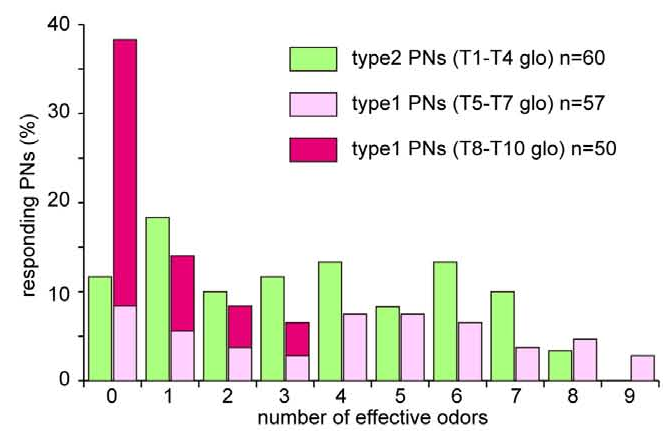

C

D

medial view

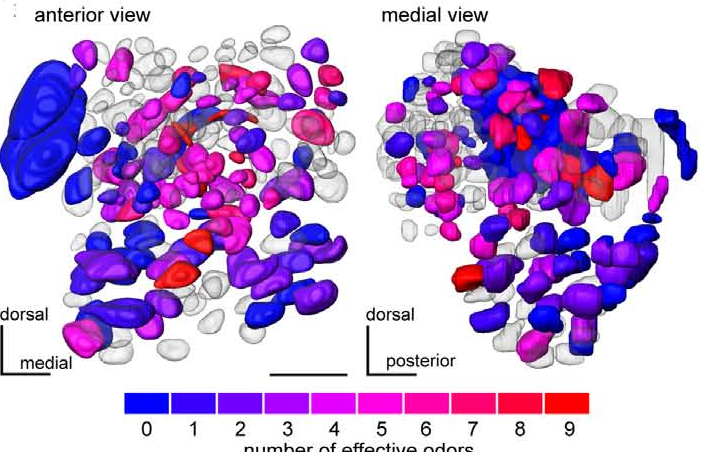

E

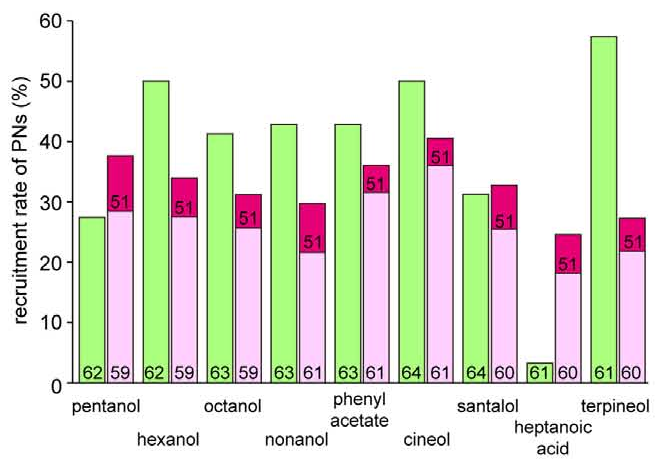

$\square$ type2 PNs (T1-T4 glo)

$\square$ type1 PNs (T5-T7 glo)

type1 PNs (T8-T10 glo)

type2 PNs (T1-T4 glo)

type1 PNs (T5-T7 glo)

type1 PNs (T8-T10 glo)

sex-pheromone PNs

ALT PNs

FIGURE 3 | Classification of PNs according to odor-specificities. (A) Response intensities to nine tested odorants in recorded PNs. We summarized response intensities to nine odorants, PN types, innervating glomeruli and odor spectra groups of 178 identified PNs. Response intensities are colored according to the values of "R-Ro" values (see "Materials and Methods"). PNs are classified into five odor spectra groups by cluster analyses using Ward's method (Supplementary

Figure S1). Post-recording visualization revealed innervating glomeruli and PN types. Innervating glomeruli are identified based on the 3D-map of the cockroach AL

(Continued) 


\section{FIGURE 3 | Continued}

(Watanabe et al., 2010). (B) Odor-specificities of PNs. A zero effective odor number means that PNs did not show any excitatory responses to all tested nine odors. The percentages of responding PNs were calculated in each of pathways (type2 PNs, green bars, $n=60$; type1 PNs, red bars, $n=107$ ). Within type1 PNs, PNs arborizing in the T8-T10 group glomeruli (dark red bars, $n=50$ ) show higher odor-specificity than those arborizing in the T5-T7 group glomeruli (light red bars, $n=57$ ). (C,D) Glomerular maps of odor-specificities, viewed anteriorly (C) and medially (D). Glomeruli innervated by recorded PNs are colored according to the number of effective odors. The colder color represents higher odor-specificity. When PNs innervated the same glomerulus and exhibited different effective odor numbers, we colored the glomerulus based on the largest effective odor number. (E) Recruitment rates of PNs. The percentages of responding type2 PNs (green bars) and type1 PNs (red bars) per odor are shown as recruitment rates. Numbers denoted in bars are sample numbers used in the analysis.

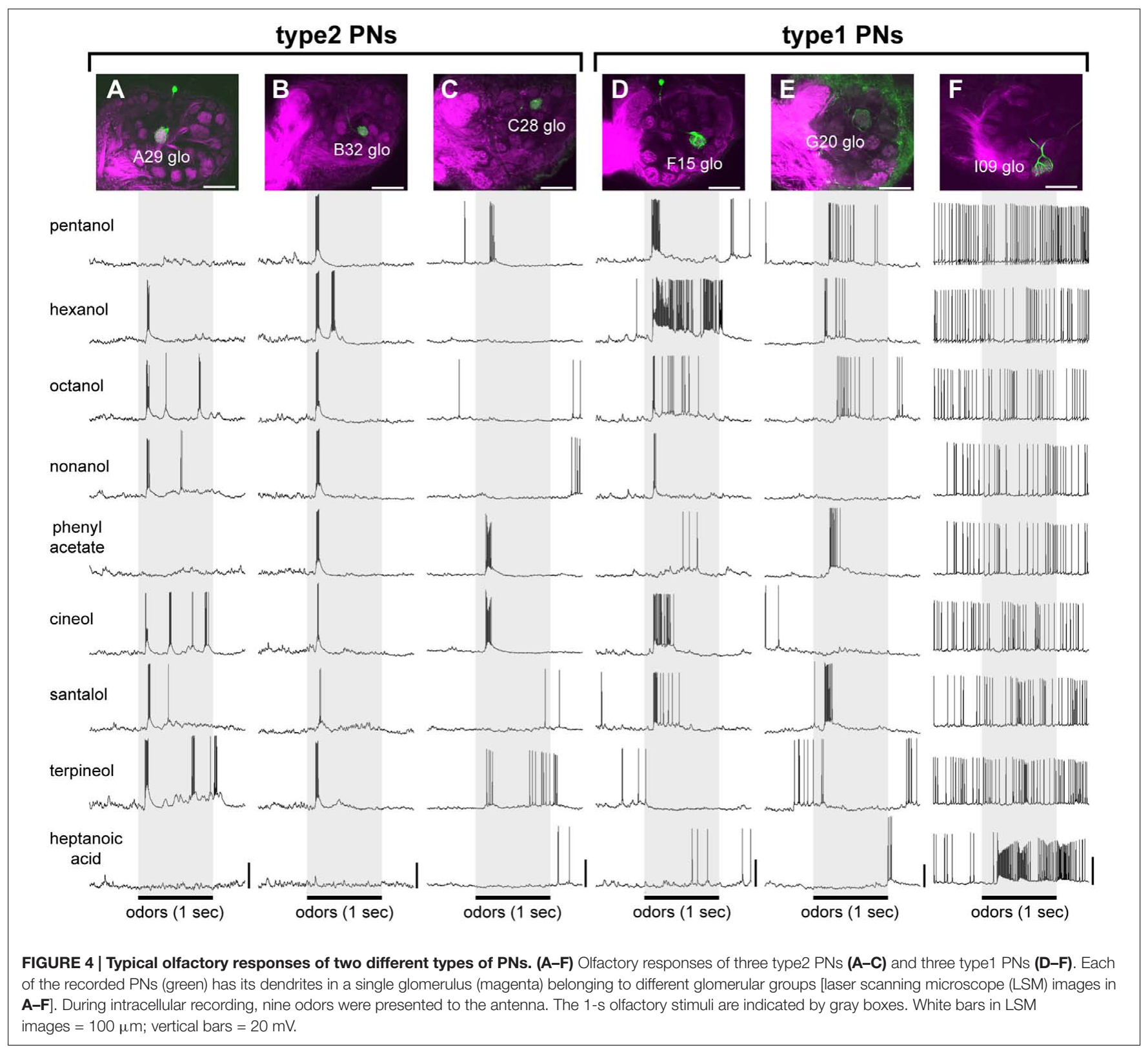

was represented as a peri-stimulus time histogram (PSTH) with a bin of $20 \mathrm{~ms}$. We classified odor-induced PSTHs into several clusters based on the cluster dendrogram. Using the "clusplot" package in R software, we plotted scores of the first two principal components (PCs) obtained from PCA. Distributions of the first two PCs are shown as box plots and variances of PCs were statistically compared using the $F$-test or two-way ANOVA test.

Cluster analysis using PSTHs strongly revealed two different odor-induced on-phasic responses in type2 PNs. To evaluate odor-induced on-phasic responses in type2 PNs in greater detail, we used 160 responses from 16 different type2 PNs that exhibited 


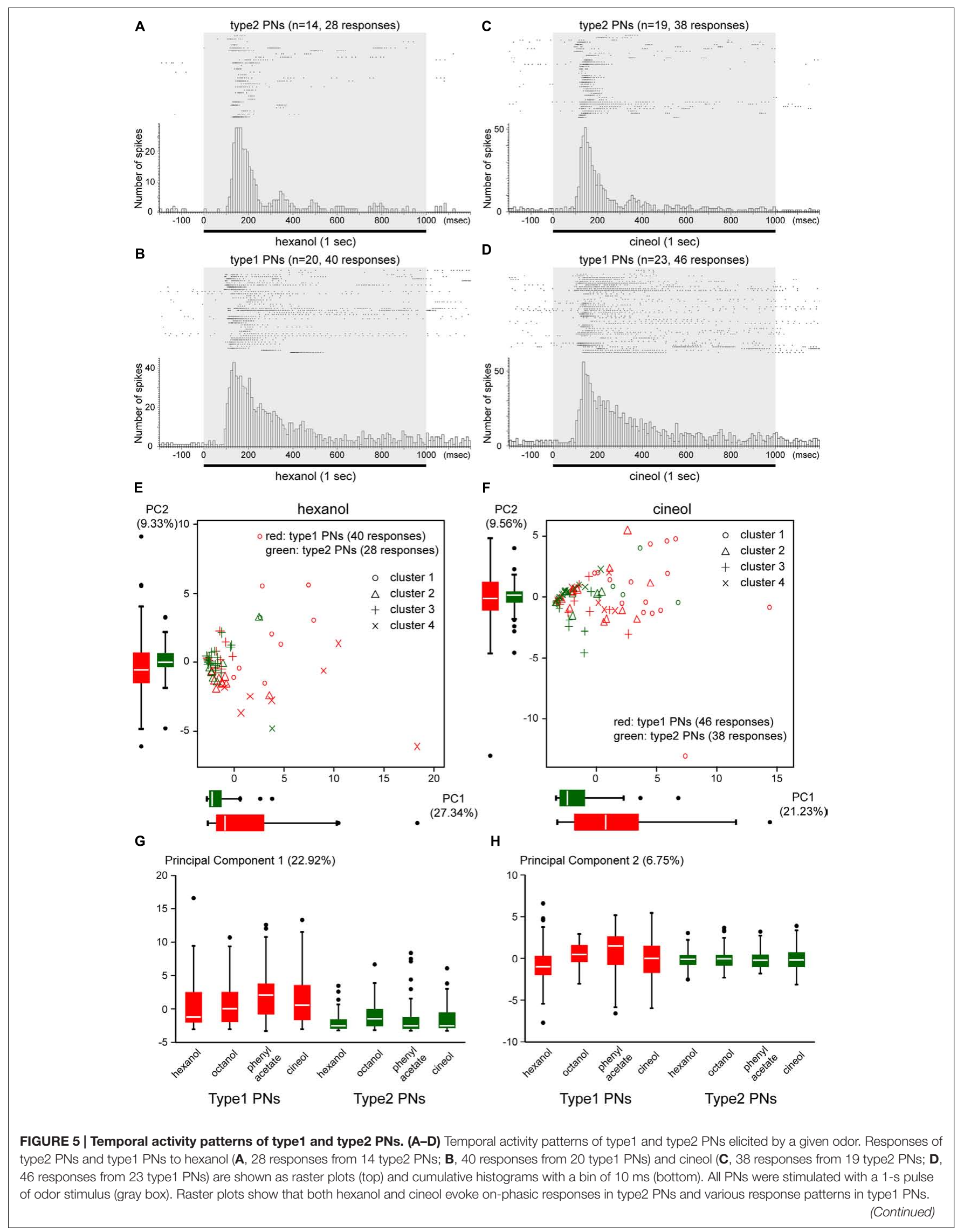




\section{FIGURE 5 | Continued}

(E,F) Differences in temporal activity patterns between type1 and type2 PNs. Based on the peri-stimulus time histograms (PSTHs) with a bin of $20 \mathrm{~ms}, 68$ responses to hexanol (E) and 84 responses to cineol (F) are, respectively, clustered into four clusters (Supplementary Figures S2, S3). Responses of type1 and type2 PNs are, respectively, plotted red and green using the first two PCs (PC 1 and PC 2). Distributions of PC 1 and PC 2 in type1 and type2 PNs are shown as box plots. Each marker represents the response clusters (Supplementary Figures S2, S3). PC 1 and PC 2 explain $36.67 \%$ of the point variability in (E) and $30.79 \%$ in (F). $(\mathbf{G}, \mathbf{H})$ Differences of temporal activity patterns across odors. Based on PSTHs of 284 responses to four different odors, we performed PCA. The distributions of the first two PCs (PC 1, G; PC 2, H) are displayed as box plots. The PC 1 and PC 2 explain 22.92 and $6.75 \%$ of the variability of responses, respectively. In (E-H), the line in the box and the box represents the median and the quartiles, respectively. Outliers are shown as dots.

excitatory responses to more than five different odors. Olfactory responses are shown as time-courses of instantaneous spike frequencies. The instantaneous spike frequency was defined as reciprocals of time intervals between successive spikes. In each olfactory response, we identified a spike that a time-course of instantaneous spike frequencies peaked or plateaued, which was termed a "peak spike". Because the odor arrival time to antenna varied (10-20 ms) across specimens, the timing of each peak spike was corrected as follows; " $t_{\text {peak }}-t_{\text {first }}$ ", where $t_{\text {peak }}$ was a time of the peak spike from the odor onset (timings of opening of solenoid valves) and $t_{\text {first }}$ was a time of earliest odor-induced spike in the specimen. Peak spikes were classified into three groups based on its instantaneous spike frequencies: $>300 \mathrm{~Hz}, 200-$ $300 \mathrm{~Hz}$, and $<200 \mathrm{~Hz}$. One-way ANOVA and post hoc Tukey test was used to compare time distribution patterns of peak spikes in the three groups.

Using the standard glomeruli map in the male cockroach $\mathrm{AL}$ (Watanabe et al., 2010, 2012b), we colored glomeruli based on anatomical and physiological properties of innervating PNs.

\section{RESULTS}

\section{Segregated Parallel Pathways from the Periphery to the Higher Brain Centers}

In the cockroach, axons of uniglomerular PNs run through the $\mathrm{m}$-ALT and nearby tracts (ALT-2 and ALT-3), and terminate in the medial and lateral calyces (CA) of the MB first and then in the LH (Malun et al., 1993; Strausfeld and Li, 1999a; Nishino et al., 2012a). Retrograde staining of PNs revealed all glomeruli were innervated by PNs running m-ALT and nearby tracts in the cockroach (Figures 1A,B). Except for two sex pheromonereceptive macroglomeruli that were each innervated by multiple PNs (Nishino et al., 2011), individual ordinary glomeruli were in principle innervated by a single uniglomerular PN (Figures 1A,B; Ernst and Boeckh, 1983). PNs innervating the antero-dorsal group glomeruli (arrowheads in Figures 1A,B), and those innervating the postero-ventral group glomeruli (arrows in Figure 1B) formed two distinct axon bundles in the AL.

Based on intracellular staining and Golgi staining, several morphological types of PNs have been identified in the cockroach brain (Malun et al., 1993; Strausfeld and Li, 1999a). We identified type1 and type2 PNs based on their terminal zones in the calyces; terminal blebs of each type $1 \mathrm{PN}$ are broadly distributed within zones III and IIIA of calyces, and those of each type2 PN are concentrated to the zone I (Strausfeld and Li, 1999a; Takahashi et al., 2017). In the current study, we intracellularly stained 115 type1 PNs, 63 type2 PNs, and 11 PNs of other types. Type1 and type2 PNs have cell bodies in the antero-dorsal region of the AL (Figures 1A-D). The results revealed that axons of type2 and type1 PNs ran through separate bundles in the AL (Figures 1A-D). After running through the m-ALT, axons of type1 and type2 PNs turned laterally in the anterior side of the $\mathrm{MB}$ pedunculus, and finally terminated in the MB calyces and the LH (Figures 1C,D). Based on intracellular staining results, we mapped 106 glomeruli with respect to innervating PN types (Figures 1E,F). Type2 and type1 PNs had dendrites in the antero-dorsal and postero-ventral group glomeruli, respectively (Figures 1E,F). Among the 106 glomeruli analyzed, only 10 postero-ventral group glomeruli were connected with the ALT-2 or ALT-3 PNs previously identified (Malun et al., 1993), and two macroglomeruli were connected with sex pheromone-sensitive PNs that terminated in specific regions of higher brain centers (Nishino et al., 2011, 2012a). Here, we focused on the two major streams formed by type1 and type2 PNs.

Dual intracellular staining unambiguously revealed that axon terminals of type1 PNs were spatially segregated from those of type2 PNs not only in the MB calyces, but also in the LH (Figures 2A-D). Type1 PNs terminated in zones III and IIIA of the MB calyces and the central region of the LH (Figures 2A-D). Conversely, type2 PNs terminated in the peripheral-most region of the calyces (zone I) and the antero-dorso-lateral region of the LH (Figures 2A-D). Segregation of axon terminals between type1 and type2 PNs was observed in all dual intracellular staining specimens $(n=5)$. In previous cockroach studies, OSNs in antennal perforated basiconic sensilla were reported to terminate specifically in the antero-dorsal group glomeruli, whereas those in the trichoid and grooved basiconic sensilla terminated in postero-ventral group glomeruli (Figure 2E; Watanabe et al., 2012b). Thus, type1 and type2 PNs appear to receive sensory inputs from different types of antennal sensilla (Figure 2E). The current results suggested that general odors are processed by distinct parallel olfactory pathways from the periphery to the higher brain centers in the cockroach brain (Figure 2E).

\section{Odor-Specificities of Two Different Types of PNs}

We successfully recorded intracellular responses to nine odorants in 184 PNs (including those innervating the same glomerulus). Among them, we unambiguously identified glomeruli with dendrites of 60 type2 PNs, 107 type1 PNs, and 11 other types of PNs (Figure 3A). In each recording, we calculated response intensities to nine odorants by subtracting the number of spikes during the 1-s pre-stimulation period $\left(\mathrm{R}_{0}\right)$ from the number 


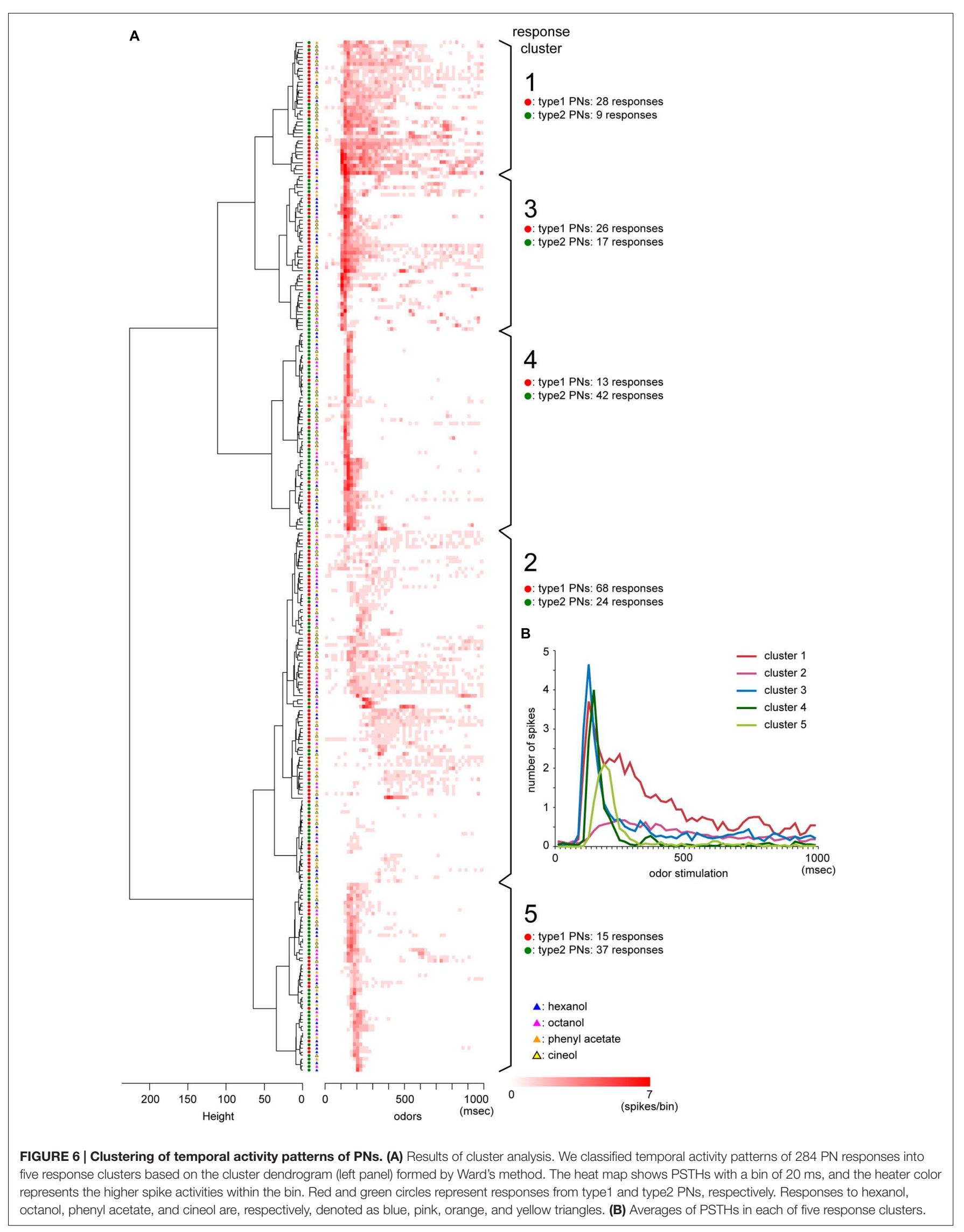




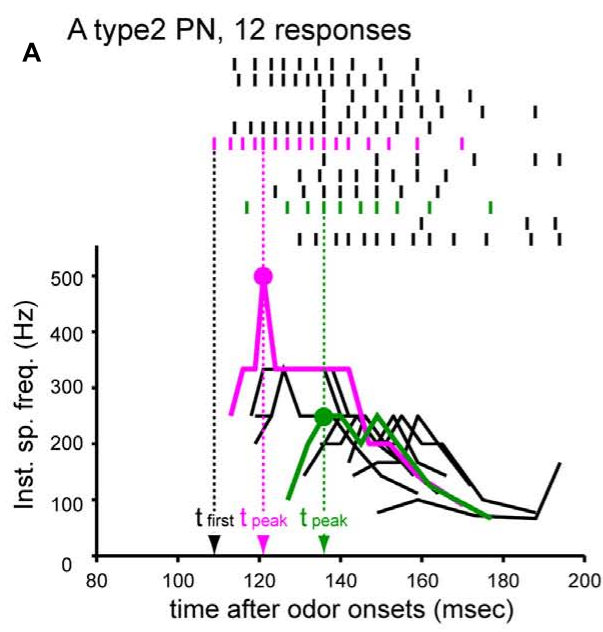

D

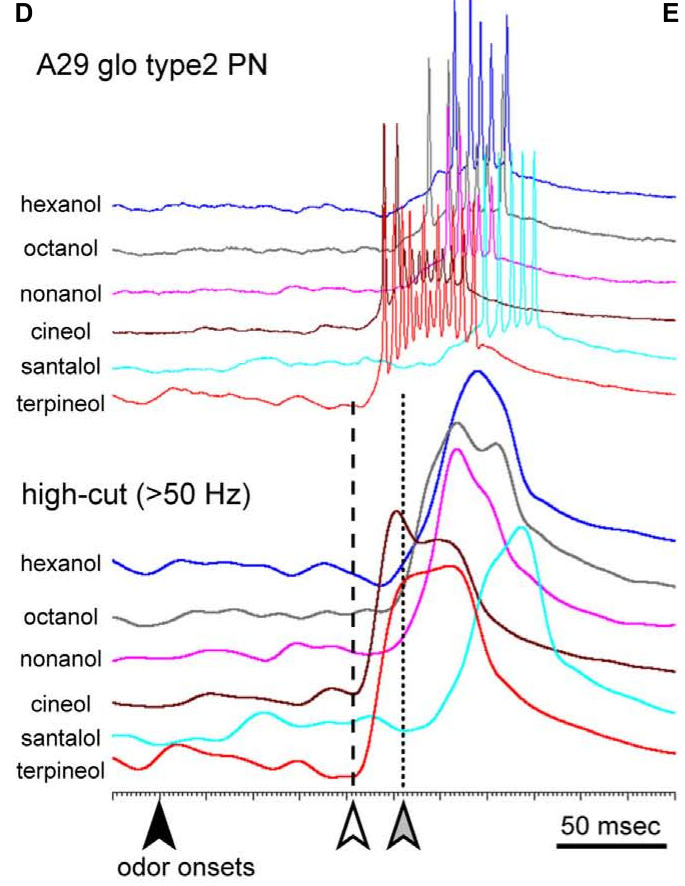

16 type2 PNs, 160 responses

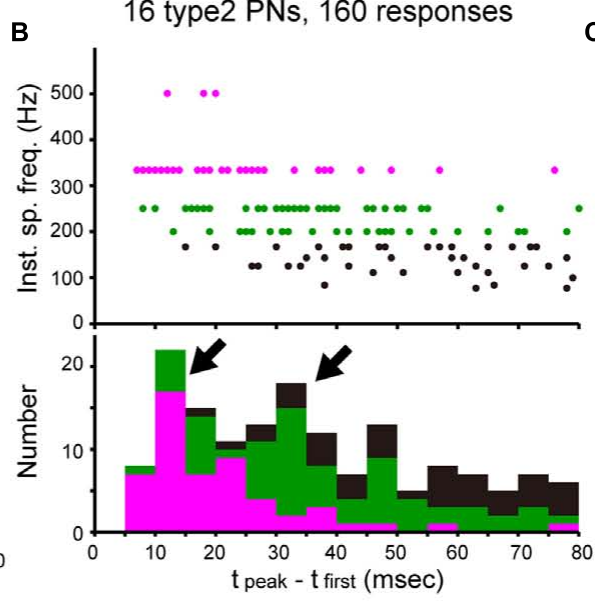

C
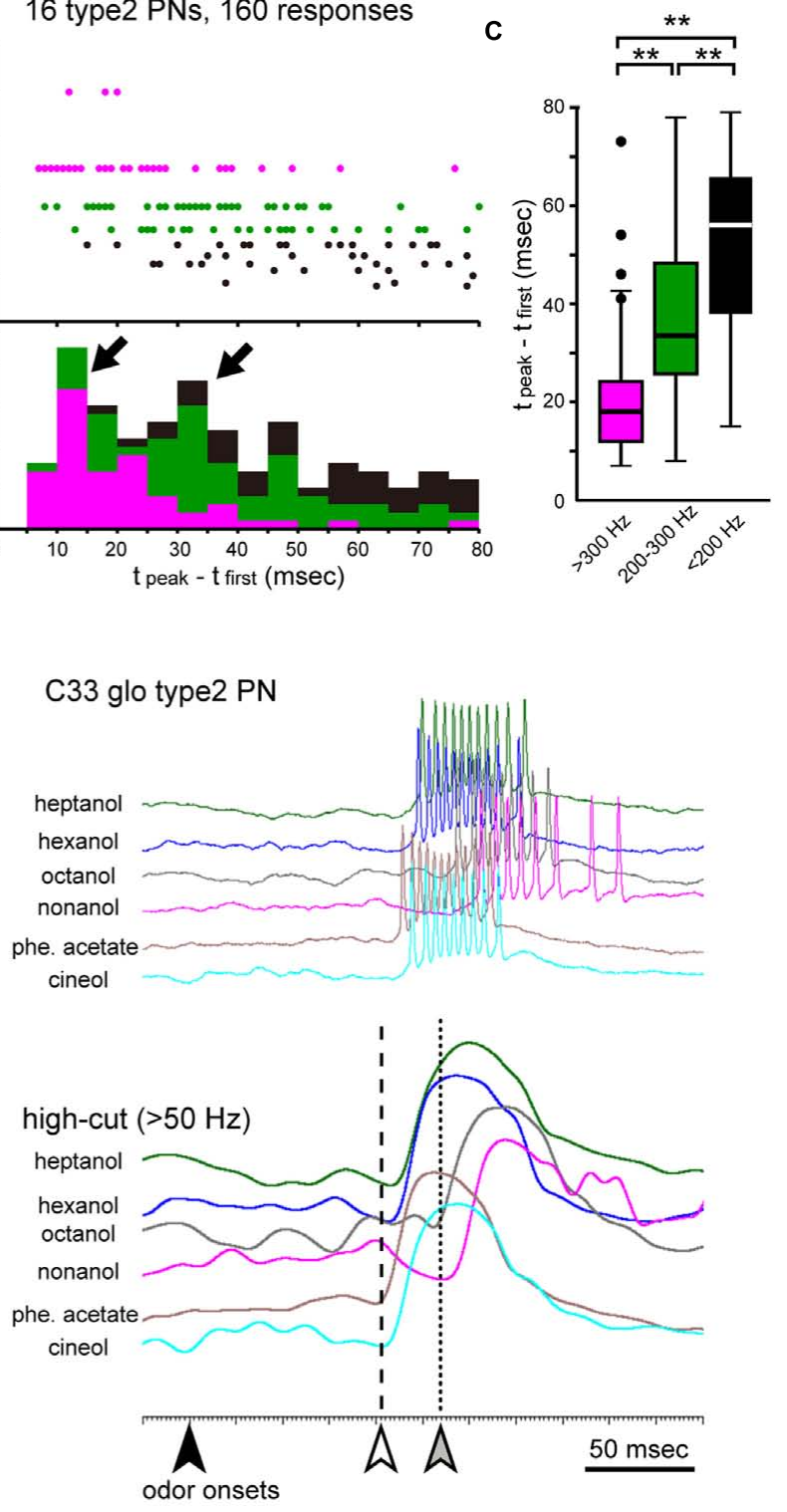

F

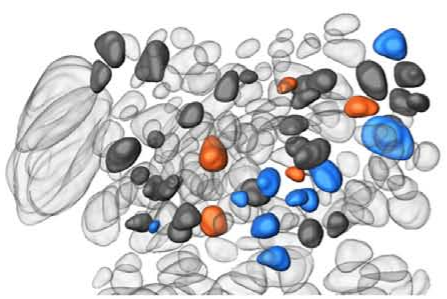

octanol

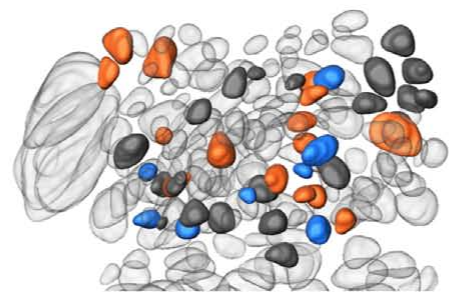

cineol

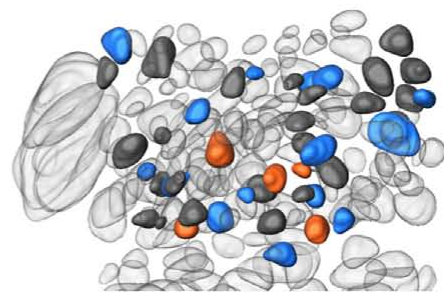

terpineol

early response

late response

no response

FIGURE 7 | Early and late responses in type2 PNs. (A) Temporal dynamics in odor-induced action potentials of a type2 PN. On-phasic responses to six different odors (12 responses) are shown as raster plots (top) and time-courses of instantaneous spike frequencies (bottom). In each response, we identified a "peak spike" (dots; see "Materials and Methods"). Magenta and green responses indicate typical early and late responses, respectively. (B,C) Time distribution of peak spikes obtained from 160 responses. We identified peak spikes from 160 olfactory responses recorded from 16 different type2 PNs. The timing of each peak spike is corrected as follows; $t_{\text {peak }}-t_{\text {first }}$, where $t_{\text {peak }}$ is a time of the peak spike from odor onset and $t_{\text {first }}$ is a time of earliest odor-induced spike in the specimen 


\section{FIGURE $7 \mid$ Continued}

(exampled in A). A total of 160 peak spikes are plotted in a scatter diagram (upper in $\mathbf{B}$ ), and the instantaneous spike frequencies ranged from $>300 \mathrm{~Hz}$, 200-300 Hz, and <200 Hz, which are colored as magenta, green, and black dots, respectively. In the histogram (bottom in B), the number of peak spikes are counted every 5 ms after $t_{\text {first }}$, and there are two prominent peaks of the histogram (arrows in $\mathbf{B}$ ). Peak spikes with high instantaneous spike frequencies (>300Hz; magenta boxes in $\mathbf{B}, \mathbf{C}$ ) are distributed significantly earlier than those with low instantaneous spike frequencies (200-300 Hz; green boxes in $\mathbf{B}, \mathbf{C})$. In (C), the line in the box and the box represents the median and the quartiles, respectively. Outliers are shown as dots. Results of statistical comparison using ANOVA and post hoc Tukey test are shown as asterisks ( $t$-test, $\left.{ }^{* *} P<0.01\right)$. (D,E) Olfactory responses of two different type2 PNs. To show rising points of early (white arrowheads) and late responses (gray arrowheads), excitatory responses elicited by different odor stimuli (top) are processed by a low-pass filter set at $50 \mathrm{~Hz}$ (bottom). Olfactory responses are arrayed based on odor onsets (black arrowheads). (F) Response type-specific glomerular organization. Glomeruli innervated by recorded type2 PNs are colored according to response types. When type2 PNs exhibited early or late responses to a given odor (octanol, cineol, or terpineol), glomeruli innervated by the PNs are colored orange or blue, respectively. Glomeruli innervated by PNs that did not show any excitatory responses to the odor are colored gray.

of spikes during the $1 \mathrm{~s}$ odor stimulation (R). Endogenous spike activity of each type $2 \mathrm{PN}$ was nearly silent (average: $1.94 \mathrm{~Hz} ; 60$ type2 PNs; $\mathrm{R}_{0}, n=1077$ ), whereas type1 PNs exhibited spontaneous spike activities of various frequencies (average: $6.30 \mathrm{~Hz} ; 107$ type1 PNs; $\mathrm{R}_{0}, n=1926$ ). Therefore, type1 PNs exhibited not only excitatory, but also inhibitory responses to odors (blue colors in Figure 3A). We performed cluster analysis using response intensities to nine odorants, and classified recorded PNs into five different odor spectra groups (Figure $\mathbf{3 A}$ and Supplementary Figure $\mathbf{S 1}$ ). The "one glomerulus - one PN" relationship is applicable to the cockroach (Boeckh and Ernst, 1987; Figures 1A,B), and PNs originating from the same glomerulus tended to belong to the same odor spectra group (Figure 3A). Therefore, response properties of PNs were conserved among individuals. Among five odor spectra groups, group 2, which exhibited inhibitory responses to nine tested odors, and group 5, which exhibited strong excitatory responses to many odors, were predominantly composed of type 1 PNs. Thus, type 1 and type2 PNs tend to belong to different odor spectra groups ( $\chi^{2}$-test, $P=9.95 \times 10^{-8}, d f=4, \chi^{2}=38.25$ ).

Odor-specificities of both PN types were estimated by the number of effective odors (Figures 3B-D). The result revealed that $48 \%$ of recorded type 2 PNs $(n=60)$ exhibited excitatory responses to more than four different odors out of nine tested odors. Conversely, $38 \%$ of recorded type1 PNs $(n=107)$ exhibited no responses to nine tested odors. On average, a single type $2 \mathrm{PN}$ was activated by approximately 3.39 of the nine test odors (median, 3), whereas a single type1 PN on average responded to approximately 2.48 of the test odors (median, 1). These results suggested that type 2 PNs were significantly broader tuned than type1 PNs to the nine tested odors $\left(\chi^{2}\right.$-test, $\left.P=2.26 \times 10^{-3}, d f=9, \chi^{2}=25.29\right)$. Additionally, type1 PNs exhibited different odor-specificities depending on innervating glomerular groups (Figures 3B-D). On average, 61\% of recorded type1 PNs innervating the T5-T7 group glomeruli exhibited excitatory responses to more than four different odorants $(n=57$; average, 4.07 ; median, 4 ), whereas $64 \%$ of recorded type1 PNs innervating the T8-T9 group glomeruli exhibited no responses to nine tested odors ( $n=50$; average, 0.63 ; median, 0$)$. These results suggested that odor-specificities of type1 PNs innervating the T8-T10 group glomeruli were significantly higher than type1 PNs innervating the T5-T7 group glomeruli $\left(\chi^{2}\right.$-test, $\left.P=5.99 \times 10^{-7}, d f=9, \chi^{2}=46.01\right)$.

Next, we quantified all PNs activated by each tested odor and calculated the recruitment rates of type1 and type2 PNs to aiven odor stimulus (Figure 3E). Generally, a given odor recruited both type 1 and type 2 PNs. The average proportion of activated type 2 PNs (38.47\%) was not significantly different from type1 PNs (32.86\%) (paired $t$-test, $P=0.278, d f=8, T=1.16$ ). However, from the view-point of each odor, the recruitment rates between type 1 and type 2 PNs varied ( $\chi^{2}$-test, $P=3.31 \times 10^{-5}$, $d f=8$, $\left.\chi^{2}=34.49\right)$. For example, heptanoic acid specifically activated type1 PNs, and there were only two type 2 PNs that exhibited excitatory responses to the odor. The result matched the spatial activation patterns of glomeruli observed in the previous imaging study (Watanabe et al., 2012a). Recruitment rates of type1 PNs innervating the T5-T7 group glomeruli (average, 26.25\%) were significantly higher than those of type1 PNs innervating the T8-T10 group glomeruli (average, 6.37\%) (paired $t$-test, $\left.P=1.15 \times 10^{-5}, d f=8, T=8.59\right)$. Moreover, type1 PNs exhibited different response profiles depending on the glomerular groups.

\section{Temporal Activity Patterns of Two Different Types of PNs}

We show olfactory responses of three type 2 PNs and three type1 PNs belonging to different glomerular groups in Figure 4. Generally, type2 PNs exhibited phasic on-responses and subsequent cessations of spike activity to effective odors (Figures 4A-C). The excitatory on-response persisted for approximately 50-100 ms. In some specimens, the excitatory phase and the break phase alternately appeared during the stimulus period (Figures 4A,B). In each type2 PN, different olfactory stimuli induced phasic on-responses. The excitatory on-responses were commonly observed among type2 PNs, though effective odors differed among different PNs (Figures 3A, 4A-C). In contrast to type 2 PNs, different olfactory stimuli induced different temporal activity patterns in each of the type1 PNs (Figures 4D-F). For example, type1 PNs innervating the F15 glomerulus exhibited phasic on-response to nonanol but tonic response to hexanol (Figure 4D). Olfactory responses of type1 PNs tended to be more temporally complicated compared with the phasic on-responses of type 2 PNs. In addition to excitatory responses, inhibitory responses characterized with prominent IPSPs were observed in type1 PNs that exhibited spontaneous spike activities (Figures $\mathbf{3 A}, \mathbf{4 F}$ ).

We summarized the temporal activity patterns of type 1 and type2 PNs recruited by a given odor stimulus (Figure 5). The responses to hexanol (28 responses from 14 type2 PNs; 40 responses from 20 type1 PNs) and to cineol (38 responses from 


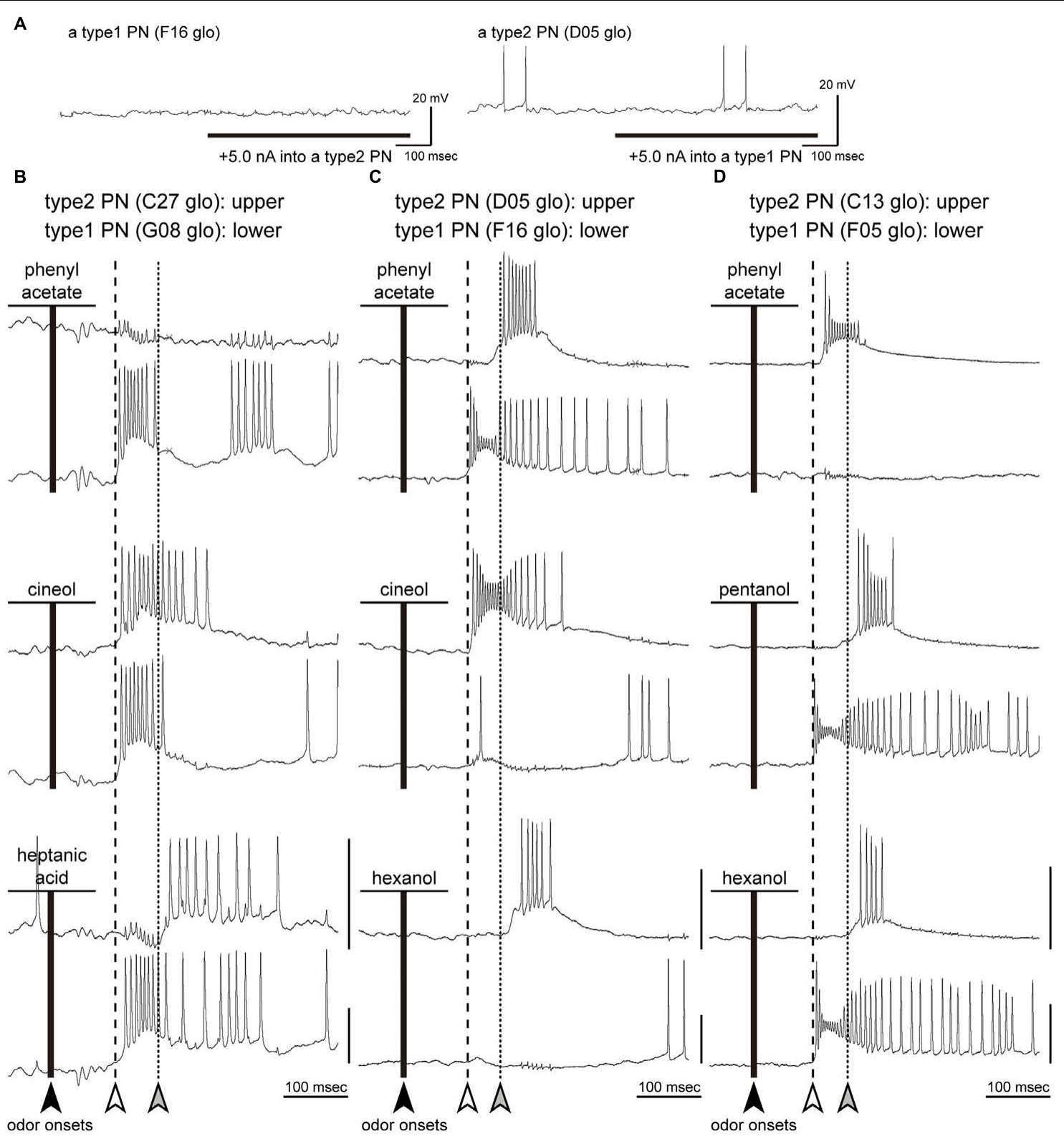

FIGURE 8 | Simultaneous intracellular recordings from a type1 PN and a type2 PN. (A) Current injection to a PN. The artificial excitation of one PN by an inward current injection $(5.0 \mathrm{nA})$ did not affect the membrane potential of the other PN. (B-D) Three pairs of response traces of simultaneous recordings from a type2 PN (upper traces) and a type1 PN (lower traces). The glomeruli innervated by PNs are denoted in each panel. We selected typical combinational responses elicited by three different odorants (black lines). Olfactory responses are arrayed based on timings of odor onsets (black arrowheads). Based on type2 PN response patterns, we identified latencies of early (white arrowheads and broken lines) and late responses (gray arrowheads and dotted lines) in each simultaneous recording. In each trace, there was considerable cross-talk (1-5 mV) between the two simultaneously recorded signals. Vertical bars $=20 \mathrm{mV}$.

19 type2 PNs; 46 response from 23 type1 PNs) are shown as raster plots and accumulated histograms in Figures 5A-D. We statistically compared histograms during the period of odor stimulation (gray bar in Figures $\mathbf{5 A - D}$ ) and revealed that a given odor elicited both type1 and type2 PNs with significantly different temporal patterns (KS-test: hexanol, $P=1.01 \times 10^{-11}$, $D=0.51$; cineol, $\left.P=1.97 \times 10^{-10}, D=0.48\right)$. In both odor stimuli, differences of temporal activity patterns between type1 and type 2 PNs reached peaks at two different timings: a period of
$100-130 \mathrm{~ms}$ and a period of $250-290 \mathrm{~ms}$ after the odor onset. The former and the later peaks appeared to reflect the differences in response latencies and durations between type 1 and type2 PNs.

To compare odor-induced temporal activity patterns across multiple PN responses, we performed cluster analyses and PCAs using PSTHs with a bin of $20 \mathrm{~ms}$ (Figures 5E,F and Supplementary Figures S2, S3). Based on cluster dendrograms, PSTHs of $68 \mathrm{PN}$ responses to hexanol and $84 \mathrm{PN}$ responses to cineol were, respectively, classified into four clusters 


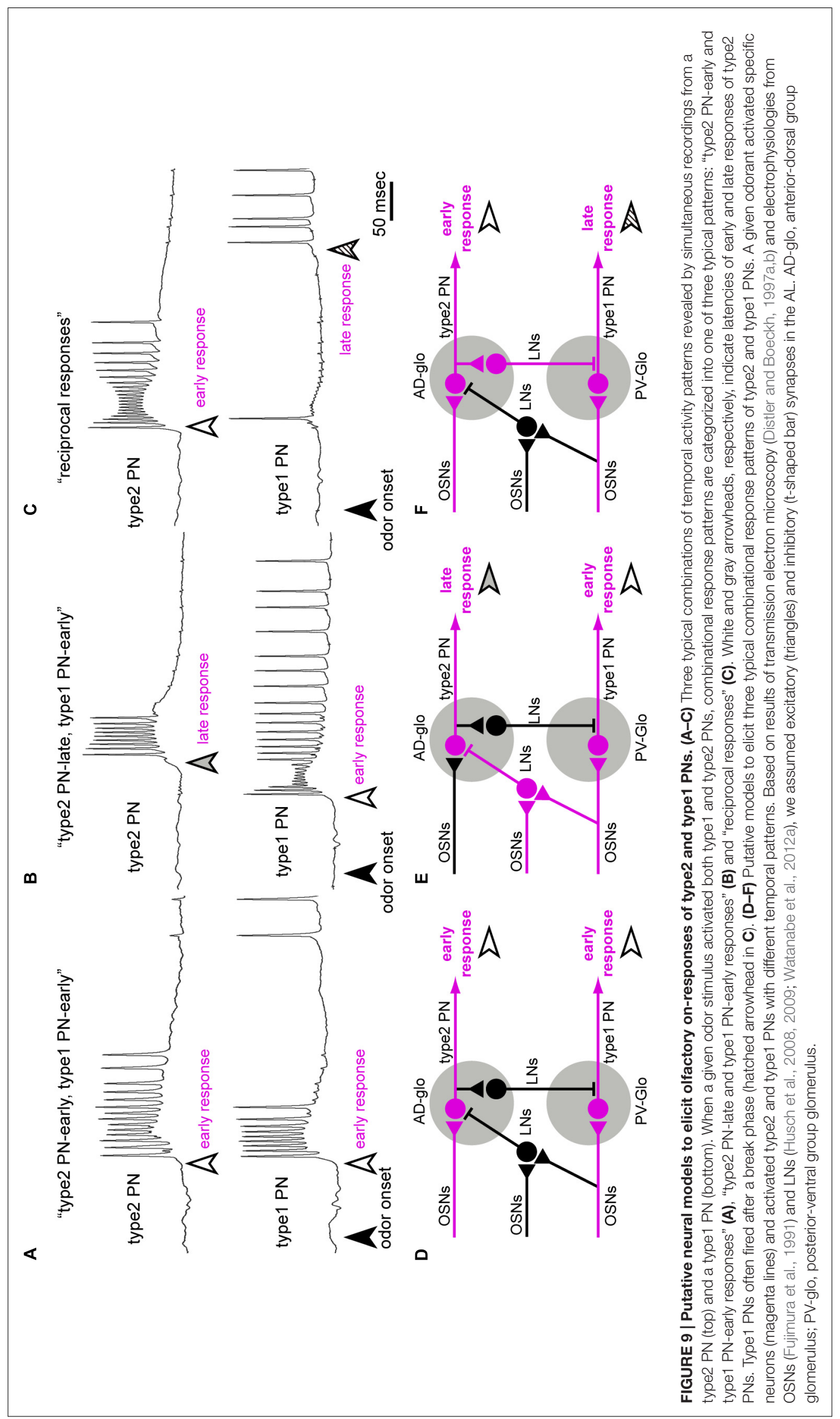


(Supplementary Figures S2, S3). In both odor stimuli, responses of type 2 PNs were grouped together into one or two clusters (hexanol, cluster 3; cineol, clusters 3 and 4), whereas those of type1 PNs were distributed throughout four clusters (Figures 5E,F and Supplementary Figures S2, S3). Consistent with cluster analysis results, the first two PCs (PC 1 and PC 2) were narrowly distributed in type2 $\mathrm{PNs}$ and broadly distributed in type1 PNs (Figures 5E,F). The PC variances were significantly different between type1 and type2 PNs (F-test; hexanol, PC $1, P=2.15 \times 10^{-6}, F=6.65$, PC $2, P=2.15 \times 10^{-6}$, $F=3.16$; cineol, PC 1, $P=0.0060, F=2.50$, PC 2, $P=0.00011$, $F=3.74$ ). These results strongly suggested that a given odor elicited similar temporal response patterns in different type2 PNs, whereas a given odor-induced temporally diverse activity patterns in different type1 PNs.

To evaluate temporal activity patterns induced by different odors, we classified $284 \mathrm{PN}$ responses induced by four different odors (hexanol, octanol phenyl acetate, and cineol) into five clusters (Figure 6). Among the five clusters, clusters 1 and 2, which showed phasic-tonic spike activities, were predominantly assigned to type1 PN responses, whereas clusters 4 and 5, which showed on-phasic spike activities, were predominantly assigned to type2 $\mathrm{PN}$ responses (Figure 6). Thus, type1 and type $2 \mathrm{PN}$ responses were clustered into different groups $\left(\chi^{2}\right.$-test, $\left.P=2.05 \times 10^{-10}, d f=4, \chi^{2}=51.18\right)$. Conversely, five response clusters were not affected by differences in odor stimuli $\left(\chi^{2}\right.$-test, $\left.P=0.11, d f=12, \chi^{2}=18.23\right)$. PCA revealed that the first two PCs (PC 1 and PC 2) were narrowly distributed in type2 PNs and broadly distributed in type1 PNs (Figures 5G,H). The PC 1 variances were significantly different across PN types, but not across odors (two-way ANOVA; odors, $P=0.319$, $d f=3$, $F=1.18$; PN types, $\left.P=4.74 \times 10^{-11}, d f=1, F=46.95\right)$. These results strongly suggested that different odors induced similar temporal activity patterns in type 2 PNs and varied temporal activity patterns in type1 PNs.

\section{Odor-Specific Early and Late Responses of Type2 PNs}

Type2 PNs generally exhibited phasic on-responses with short latencies. However, cluster analysis of PSTHs suggested that phasic on-responses of type2 PNs were further classified into two different temporal patterns: clusters 4 and 5 in Figure 6A. Averages of PSTHs in each cluster revealed that responses in cluster 4 exhibited stronger spike activities with earlier latencies than those in cluster 5 (Figure 6B). To describe temporal activity patterns of type 2 PNs in more detail, we analyzed odorinduced spike arrays in 160 phasic on-responses obtained from 16 different type 2 PNs that exhibited excitatory responses to more than five different odors (Figures 7A-C). In each response, we identified a spike at which instantaneous spike frequencies peaked or plateaued, which was termed a "peak spike" (dots in Figure 7A). Time distribution of 160 peak spikes revealed that the phasic on-responses of type 2 PNs were classified into two types: early and late responses (arrows in Figure 7B), exactly corresponding to the clusters 4 and 5 (Figure 6A). Additionally, peak spikes with high spike frequencies $(>300 \mathrm{~Hz}$; magenta in Figures $7 \mathbf{B}, \mathbf{C}$ ) were distributed significantly earlier than those with low spike frequencies (200-300 Hz; green in Figures 7B,C; ANOVA and post hoc Tukey test, $\left.P=4.0 \times 10^{-7}, d f=16.25\right)$. These results also suggested that type 2 PNs exhibited early and strong response or late and weak response to a given effective odor.

Early and late responses were commonly observed in type2 PNs which exhibited excitatory responses to multiple odors, and the latencies of late responses were 30-40 ms longer than the early responses (Figures 7D,E). In each specimen, the EPSP rise was almost identical for both early and late responses elicited by different odors (lower traces in Figures 7D,E), suggesting that specific neural mechanisms underlie early and late responses in type2 PNs. In individual type2 PNs, an average of 1.9 odors induced late responses, and an average of 1.1 odors induced early responses. Thus, odor-specificity of the early response was significantly greater than that of the late response (Wilcoxon-test, $\left.N=63, P=2.8 \times 10^{-4}\right)$. In the case of a type $2 \mathrm{PN}$ innervating the A29 glomerulus (Figure 7D), two odors (cineol and terpineol) elicited early responses (white arrowhead in Figure 7D), and four odors (hexanol, octanol, nonanol, and santalol) induced late responses (gray arrowhead in Figure 7D). In contrast, a type2 PN with dendrites in the $\mathrm{C} 33$ glomerulus exhibited early responses to heptanol, hexanol, phenyl acetate, and cineol (white arrowhead in Figure 7E), and exhibited late responses to octanol and nonanol (gray arrowhead in Figure 7E). Thus, the response type

TABLE 1 | Anatomical and physiological differences between type1 and type2 projection neurons (PNs).

Type 2 PNs
Antero-dorsal group glomeruli
T1-T4 groups

Perforated basiconic sensilla

Antennal sensilla

Output

Mushroom bodie (MB) calyces

Lateral horn (LH)

Response properties

Temporal pattern zone I

Antero-dorso-lateral region

low

On-phasic
Odor-specificity
Type 1 PNs

\begin{tabular}{ll}
\multicolumn{2}{c}{ Postero-ventral group glomeruli } \\
T5-T7 groups & T8-T10 groups \\
Trichoid sensilla & Grooved basiconic sensilla
\end{tabular}

zones III and IIIA

Central region

low

high
Various

Late response 
evoked by a given odorant differed depending on the innervating glomerulus (Figure 7F). Therefore, our results suggested that the odor-induced activity pattern of a population of type2 PNs drastically changed within a brief time window (Figure 7F).

\section{Simultaneous Intracellular Recordings from Two Different PNs}

Intracellular recordings revealed that a given odor stimulus activated both type1 and type2 PNs with different temporal patterns (Figures 4-7). In addition, there were early and late onphasic responses in type2 PNs (Figures 6, 7). To investigate the neural mechanisms underlying temporal activity patterns of PNs, we performed simultaneous intracellular recordings from a pair of type1 and type2 PNs.

We successfully conducted simultaneous intracellular recordings from six pairs of type1 and type2 PNs. In each recording, some odors elicited different excitatory responses in both type1 and type2 PNs (Figure 8). We recorded 39 combinational excitatory responses from six pairs of type 1 and type2 PNs. Among them, Figure 8 shows the combinational response patterns induced by three different odors obtained from three simultaneous intracellular recordings. In each of the traces, considerable cross-talk (1-5 mV) between two simultaneously recorded signals was identified. In these recordings, the artificial excitation of one PN by an inward current injection $(5.0 \mathrm{nA}) \mathrm{did}$ not affect the membrane potential of the other PN (Figure 8A). This suggested that there were no direct synaptic connections between the two PNs, consistent with results from post-recording visualizations (data not shown).

Based on the response patterns of a type2 PN, we identified latencies of early responses (white arrowheads and broken lines in Figure 8) and late responses (gray arrowheads and dotted lines in Figure 8) in each simultaneous recording. When a given odor stimulus elicited excitatory responses in both type1 and type2 $\mathrm{PNs}$, the combinational response patterns were categorized into one of three typical patterns (Figures 9A-C). First, both type 1 and type2 PNs started to fire nearly simultaneously at the latency of early responses (responses to cineol shown in Figure 8B). Among the 39 combinational excitatory responses, 11 responses from $4 \mathrm{PN}$ pairs were categorized into the pattern. There were no events prior to the early responses in both PN types. Second, the type1 PN fired with an early response, then the type2 PN fired with a late response (responses to heptanoic acid in Figure 8B, to phenyl acetate in Figure 8C, and to pentanol and hexanol in Figure 8D). Among the 39 combinational excitatory responses, 18 responses exhibited the pattern. This combinational response patterns were commonly observed in all PN pairs. Interestingly, the late responses were observed only in type2 PNs. Third, type1 and type2 PNs often exhibited excitatory responses reciprocally (responses to cineol and hexanol in Figure 8C). The type1 PN did not exhibit spike activities during the firing of the type2 $\mathrm{PN}$, and then fired with a low spike frequency after finishing the type $2 \mathrm{PN}$ response. Among the 39 combinational excitatory responses, 10 responses from $4 \mathrm{PN}$ pairs were categorized into the pattern. Based on the present results and previous anatomical and physiological reports in the cockroach, we propose that neural circuits in the cockroach AL are involved in odor-induced temporal activity patterns in both types of PNs (See "Discussion," Figure 9).

\section{DISCUSSION}

Cockroaches exhibit excellent olfactory discrimination and learning capabilities (Sakura et al., 2002; Watanabe et al., 2003). Higher brain centers receive odor information from a population of PNs to discriminate odors, and to acquire and retrieve olfactory memories (Watanabe et al., 2011). In the cockroach, general odors are processed in two major types of uniglomerular PNs, type1 and type2 PNs, which have been anatomically identified from distinct termination areas in the MB calyces (Strausfeld and Li, 1999a). The current results revealed that axon terminals of type1 and type2 PNs are spatially segregated in the MB calyces, as well as in the LH. In addition, type1 and type2 PNs arborize in the postero-ventral and antero-dorsal group glomeruli, respectively. The former glomeruli specifically receive olfactory inputs from antennal perforated basiconic sensilla and the latter glomeruli from trichoid and grooved basiconic sensilla (Watanabe et al., 2012b). These results indicate that the olfactory system of a phylogenetically basal insect, the cockroach, is anatomically segregated into two distinct parallel pathways from the peripheral sensory system to higher brain centers. Additionally, comprehensive intracellular recording revealed that type1 PNs and type2 PNs exhibited different odor-specificities in response to the nine tested odorants. In particular, quantitative analyses and simultaneous intracellular recording clearly reveled that a given odor-activated PNs in both pathways with different temporal patterns. Anatomical and physiological differences between type 2 and type1 PNs are summarized in Table 1. These results strongly suggest that two parallel pathways have different odor coding strategies.

The parallel pathways for processing general odors have been studied in hymenopteran insects, which are equipped with m-ALT and l-ALT PNs (Kirschner et al., 2006; Zube et al., 2008; Zwaka et al., 2016). In honey bees, a given odorant activates both l-ALT and m-ALT PNs, and these neurons exhibit different physiological properties according to odorspecificities and concentrations (Muller et al., 2002; Krofczik et al., 2008; Yamagata et al., 2009; Brill et al., 2013; Carcaud et al., 2015). The innervating glomeruli and projection patterns suggest that $\mathrm{m}$-ALT and l-ALT PNs in hymenopteran insects might correspond to type1 and type2 PNs in the cockroach, respectively. However, there are substantial anatomical differences between these insects. First, cockroaches lack uniglomerular PNs running through the 1-ALT. Second, OSNs in single pore-plate sensilla send axons to both PN types in honey bees (Kelber et al., 2006; Kropf et al., 2014). Third, terminal regions of the two PN types partially overlap in honey bees. To date, two parallel pathways to process general odors have been reported only in the cockroach (the current study) and hymenopteran insects (Galizia and Rössler, 2010; Martin et al., 2011). These phylogenetically distinct insect groups may have independently evolved parallel olfactory pathways, and may be an example of convergent evolution. 
Future studies examining olfactory pathways in other insects are needed to confirm this hypothesis.

\section{Response Properties of Each PN Type in the Cockroach Brain}

We showed that odor-specificities of type1 PNs to nine tested odorants were higher than in type2 PNs. We selected nine odorants, each eliciting strong excitatory effects in one of the eight OSN groups (Fujimura et al., 1991). Among these odorants, seven were putative ligands of perforated basiconic sensilla. Therefore, it is not surprising to find that type2 PNs, which receive sensory inputs from OSNs in the basiconic sensilla, were broadly tuned to tested odorants. Conversely, santalol and heptanoic acid are selective ligands of the grooved basiconic sensilla, and OSNs in the sensilla tend to terminate in T8-T9 group glomeruli, in which narrowly tuned type1 PNs arborize (Watanabe et al., 2012b). Thus, response ranges of PNs are strongly affected by sensory inputs from OSNs. However, specific ligands of perforated basiconic sensilla, such as low molecular alcohols, activate not only type2 PNs but also type1 PNs and vice versa. This suggests that $\mathrm{PNs}$ might be more broadly tuned to odors than their presynaptic OSNs, as reported in Drosophila $\mathrm{AL}$, indicating that the cockroach and Drosophila AL may share a similar form of lateral excitation through the recurrent pathways via local neurons (Wilson et al., 2004).

Interestingly, results showed that type1 PNs arborizing in T5-T7 group glomeruli are broadly tuned to nine tested odors, including PNs that responded to all tested odors. In the cockroach, the T5-T7 group glomeruli receive sensory inputs from trichoid sensilla (Watanabe et al., 2012b), and a part of trichoid sensilla contain OSNs that respond to the onset and offset of odor stimulus (Hinterwirth et al., 2004; Burgstaller and Tichy, 2011). This suggests that type1 PNs process more specific aspects of odor information compared with type2 PNs. Among the nine tested odors, the heptanoic acid selectively activated type1 PNs (Figure 3B). In the cockroach, behavioral repercussions of the heptanoic acid remain unclear, but some types of fatty acids, such as butyric acid, hexadecanoic acid, and pentadecanoic acid, exhibit attractive effects (Imen et al., 2015). In addition, type1 PNs with dendrites in T10 glomeruli exhibited excitatory responses to temperature and humidity changes (Nishino et al., 2003). M-ALT PNs in hymenopteran insects, which are analogs of type1 PNs in the cockroach, include PNs that process specific odors, such as cuticular hydrocarbons in ants (Nishikawa et al., 2012) and blood pheromones in honey bees (Carcaud et al., 2015). It was recently shown that m-ALT PNs are critical for successful appetitive olfactory learning (Carcaud et al., 2016).

In each type2 $\mathrm{PN}$, effective odor stimuli elicited on-phasic responses with different latencies: early and late responses. In each recording, the temporal structures of early (or late) responses elicited by different odorants were almost identical. This finding suggests that individual type2 PNs are difficult to encode odor qualities in the temporal response patterns. Conversely, cluster analysis revealed latencies and temporal activity patterns of early and late responses remained consistent among different type2 PNs. This pattern suggests that a given odor stimulus might activate many different type2 PNs with short or long latencies, and odor quality might be encoded as the extent of synchronous activity of populations of type2 PNs. Because the MB intrinsic neurons (KCs) have been reported to detect synchronized inputs from PNs in the cockroach (Demmer and Kloppenburg, 2009), inputs from type 2 PNs may be more suitable for exciting KCs compared to those from type 1 PNs.

In contrast, type1 PNs exhibited long-lasting responses during the odor stimulus period, and different odor stimuli induced different response latencies and different temporal activity patterns in each type1 PN. Thus, individual type1 PNs may encode odor-specificities in their temporal activity patterns. In fact, odor-specificity in type1 PNs, especially type1 PNs that innervate the T8-T10 glomerular groups, are higher than that in type2 PNs. In this study, we performed single-cell based analyses to reveal similarities and diversities of temporal activity patterns across PNs. However, the functional meaning of the temporal activity patterns remains unknown. PN populationbased analyses are needed in the future to reveal the differences in parallel coding strategies in the cockroach brain.

\section{Putative Neural Models of Early Olfactory Processing in the Cockroach AL}

In simultaneous recordings, we identified three typical combinational odor-induced activity patterns between a type1 PN and a type2 PN: "type2 PN-early and type1 PN-early responses," "type2 PN-late and type1 PN-early responses," and "reciprocal responses" (Figures 9A-C). Previous extracellular recordings revealed that odors used in this study elicit phasictonic spike activities in OSNs in the cockroach; each OSN exhibits a strong phasic on-response and a prolonged weak tonic response that outlasts the period of effective odor stimulation (Fujimura et al., 1991; Prof. Yokohari, personal communications). Based on these results and previous anatomical and physiological studies in the cockroach (Ernst and Boeckh, 1983; Distler and Boeckh, 1997a,b; Husch et al., 2008, 2009; Watanabe et al., 2012a), we propose a neural model of the mechanisms underlying on-responses of both PN types (Figures 9D-F). In the "type2 PN-early and type1 PN-early responses," early responses of both PNs arise at the same latencies to a given odor, with no neural events before the early responses. Thus, we hypothesize that early responses in both PN types are elicited by direct inputs from activated OSNs (Figure 9D).

In simultaneous recording, we often observed reciprocal interactions between type1 and type2 PNs that may be mediated by neural circuits interconnecting the two pathways in the AL. The "type2 PN-late and type1 PN-early responses" were observed in all pairs of type1 and type2 PNs (Figure 8). We occasionally observed putative hyperpolarizing membrane potential before the onset of the late response of the type2 PNs. This strongly suggests that inhibitory neural circuits in the cockroach $\mathrm{AL}$ might mediate late responses of type2 PNs. Interestingly, the late responses were observed only in type2 PNs. Therefore, we hypothesize that the feed-forward inhibitory pathways, which receive olfactory inputs from OSNs in the postero-ventral group glomeruli and terminate at the antero-dorsal group glomeruli 
(Figure 9E). Previous cockroach studies reported that more than $90 \%$ of LNs exhibit multiglomerular projections, with dendritic arborizations in most, but not all glomeruli, termed LN1s (Distler and Boeckh, 1997b; Husch et al., 2008, 2009; Watanabe et al., 2012a). Almost all LN1s are GABAergic, and make synapses with both OSNs and PNs (Distler and Boeckh, 1997b). In a previous study, simultaneous recordings of two different LN1s revealed that LN1s exhibit excitatory on-responses to effective odors, and odor-induced spikes are temporally synchronized between two LN1s (Watanabe et al., 2012a). Since more than 25 GABAergic LN1s are assumed to converge onto a glomerulus in the cockroach (Distler and Boeckh, 1997b), synchronized firing of multiple LN1s induced strong inhibitory effects on postsynaptic neurons. Based on the previous findings, we hypothesize that late responses of type2 PNs are mediated by synchronized firing of multiple GABAergic LN1s in the cockroach AL.

In addition, in response to a given odor, a type $1 \mathrm{PN}$ often exhibit hyperpolarization during activation of a type2 $\mathrm{PN}$, a phenomenon we refer to as "reciprocal responses" (Figure 9F). This suggests that type1 PNs might receive inhibitory inputs from activated type2 PNs. Because there are no direct connections between type1 and type2 PNs in the cockroach AL, "reciprocal responses" might be mediated by LNs. After the break phase, type1 PNs fired with low spike frequencies, and they might be induced by the post inhibitory rebound excitation and/or prolonged inputs from activated OSNs. In the cockroach AL, several LN types, except for LN1s, have been physiologically and anatomically identified (Husch et al., 2009; Fusca et al., 2015). To better understand the olfactory processing pathways in the cockroach AL, simultaneous intracellular recordings should be performed from a PN and a LN.

The current findings raise the question of what odor stimulus parameters are extracted by PNs. It remains unknown, although two different response phases of type2 PNs may provide valuable insights. If our hypothesis is true, the early responses might be driven by direct excitatory inputs from OSNs, whereas the late responses are mediated by feed-forward pathways. Thus, the latency and strength of early responses appear to reflect the timing and concentration of odor stimuli coded by OSNs, respectively. In contrast, feed-forward pathways through multiglomerular LNs integrate sensory inputs from many OSNs, suggesting that late responses of type 2 PNs might be better suited for processing of odor mixture. In fact, in each type $2 \mathrm{PN}$, odor-specificity of the late response was lower than in the early response.

It remains to be determined how do cockroaches process olfactory information from type1 and type 2 PNs in the higher brain centers. In the cockroach, KCs require convergent and synchronous inputs from multiple PNs for firing (Demmer and Kloppenburg, 2009). Additionally, inputs from PNs to KCs are modulated by four GABAergic calycal giant neurons (CGs) with dendrites in the termination fields of $\mathrm{MB}$ output neurons (Nishino et al., 2012b; Takahashi et al., 2017). Recent anatomical evidence suggests that olfactory inputs from type1 and type 2 PNs to the MB calyces are modulated by different subset of CGs (Takahashi et al., 2017). Specifically, the axon terminal of three CGs, including a non-spiking neuron, are found to converge on the terminal region of type2 PNs, forming fine and complex negative-feedback circuits. It has been reported in the locust Schistocerca americana that a non-spiking GABAergic feedback neuron provides powerful and phase-locked inhibition to $\mathrm{KCs}$ in $\mathrm{MB}$ calyces, reinforcing the ability of KCs to detect coincident spikes from PN populations (Papadopoulou et al., 2011). In addition, a CG that may modulate type1 PNs is much larger than the remaining three CGs, suggesting that it receives predominately more $\mathrm{MB}$ output neurons than other CGs. These results suggest that inputs from type1 PNs to KCs may be influenced by MB activities, such as olfactory learning and memory. Overall, the current results suggest that the cockroach uses two parallel coding strategies for processing general odor.

\section{AUTHOR CONTRIBUTIONS}

All authors had full access to all the data in the study and take responsibility for the integrity of the data and the accuracy of the data analysis. Study concept and design: HW. Acquisition, analysis and interpretation of data: HW. Drafting of the article: HW. Critical revision of the article for intellectual content: HW, $\mathrm{HN}, \mathrm{MM}$, and FY. Obtained funding: HW, HN, MM, and FY. Administrative, technical, and material support: $\mathrm{HW}$ and $\mathrm{HN}$. Study supervision: MM and FY.

\section{FUNDING}

This research was supported by Grant-in-Aid for Young Scientists (B) from the Japan Society for the Promotion of Science; Grant number: 16K18586 (to HW), the Ministry of Education, Science, Technology, Sports and Culture of Japan; grant number: 23570087 (to HN), 16H04814 (to MM), 16K07446 (to FY) and funding from the Central Research Institute of Fukuoka University; grant number: 141031 and 167104 (to FY and HW).

\section{ACKNOWLEDGMENTS}

We thank Dr. Michiko Nishikawa for valuable discussions and technical tips on the study. We also thank Mr. Itsuro Kamimura (Maxnet Co., Ltd) for technical advice for the 3D reconstructions using Amira software.

\section{SUPPLEMENTARY MATERIAL}

The Supplementary Material for this article can be found online at: http://journal.frontiersin.org/article/10.3389/fncir.2017. 00032/full\#supplementary-material

FIGURE S1 | Cluster analysis of recorded projection neurons (PNs). We performed the cluster analysis (Ward's method) using response intensities to nine odorants. We classified 178 PNs into five odor spectra groups. The five odor spectra groups are arbitrarily grouped based on the dendrogram (left panel) and response intensities (heat map). Response intensities to nine odors were summarized as heat colors. 


\section{FIGURE S2 | Cluster analysis of temporal activity patterns elicited by}

hexanol. Based on peri-stimulus time histograms (PSTHs) during the 1-s hexanol stimulations, we classified 68 PN responses into four response clusters. The four response clusters are arbitrarily grouped based on the cluster dendrogram (left panel, height 30) formed by Ward's method and PSTHs. The heat map shows PSTHs with a bin of $20 \mathrm{~ms}$, and the heater color represents higher spike activities within the bin. Red and green circles represent the responses from type1 and type2 PNs, respectively.

\section{REFERENCES}

Boeckh, J., and Ernst, K. D. (1987). Contribution of single unit analysis in insects to an understanding of olfactory function. J. Comp. Physiol. A 161, 549-565. doi: 10.1007/BF00603661

Brill, M. F., Meyer, A., and Rössler, W. (2015). It takes two - coincidence coding within the dual olfactory pathway of the honeybee. Front. Physiol. 6:208. doi: 10.3389/fphys.2015.00208

Brill, M. F., Rosenbaum, T., Reus, I., Kleineidam, C. J., Nawrot, M. P., and Rössler, W. (2013). Parallel processing via a dual olfactory pathway in the honeybee. J. Neurosci. 33, 2443-2456. doi: 10.1523/jneurosci.4268-12.2013

Burgstaller, M., and Tichy, H. (2011). Functional asymmetries in cockroach $\mathrm{ON}$ and OFF olfactory receptor neurons. J. Neurophysiol. 105, 834-845. doi: $10.1152 /$ jn. 00785.2010

Burrows, M., Boeckh, J., and Esslen, J. (1982). Physiological and morphological properties of interneurones in the deutocerebrum of male cockroaches which respond to female pheromone. J. Comp. Physiol. A 145, 447-457. doi: 10.1007/ BF00612810

Carcaud, J., Giurfa, M., and Sandoz, J. C. (2015). Differential combinatorial coding of pheromones in two olfactory subsystems of the honey bee brain. J. Neurosci. 35, 4157-4167. doi: 10.1523/jneurosci.0734-14.2015

Carcaud, J., Giurfa, M., and Sandoz, J. C. (2016). Parallel olfactory processing in the honey bee brain: odor learning and generalization under selective lesion of a projection neuron tract. Front. Int. Neurosci. 9:75. doi: 10.3389/fnint.2015. 00075

Demmer, H., and Kloppenburg, P. (2009). Intrinsic membrane properties and inhibitory synaptic input of kenyon cells as mechanisms for sparse coding? J. Neurophysiol. 102, 1538-1550. doi: 10.1152/jn.00183.2009

Distler, P. G., and Boeckh, J. (1997a). Synaptic connections between identified neuron types in the antennal lobe glomeruli of the cockroach, Periplaneta americana: I. Uniglomerular projection neurons. J. Comp. Neurol. 378, 307-319. doi: 10.1002/(SICI)1096-9861(19970217)378:3<307::AID-CNE1<3.0 $\mathrm{CO} ; 2-7$

Distler, P. G., and Boeckh, J. (1997b). Synaptic connections between identified neuron types in the antennal lobe glomeruli of the cockroach, Periplaneta americana: II. Local multiglomerular interneurons. J. Comp. Neurol. 383 529-540. doi: 10.1002/(SICI)1096-9861(19970714)383:4<529::AID-CNE9<3.0. $\mathrm{CO} ; 2-4$

Ernst, K. D., and Boeckh, J. (1983). A neuroanatomical study on the organization of the central antennal pathways in insects. Cell Tissue Res. 229, 1-22. doi: $10.1007 / \mathrm{BF} 00217877$

Fujimura, K., Yokohari, F., and Tateda, H. (1991). Classification of antennal olfactory receptors of the cockroach, Periplaneta americana L. Zool. Sci. 8, 243-255.

Fusca, D., Schachtner, J., and Kloppenburg, P. (2015). Colocalization of allatotropin and tachykinin-related peptides with classical transmitters in physiologically distinct subtypes of olfactory local interneurons in the cockroach (Periplaneta americana). J. Comp. Neurol. 523, 1569-1586. doi: 10.1002/cne.23757

Galizia, C. G., and Rössler, W. (2010). Parallel olfactory systems in insects: anatomy and function. Annu. Rev. Entomol. 55, 399-420. doi: 10.1146/annurev-ento112408-085442

Hildebrand, J. G., and Shepherd, G. M. (1997). Mechanisms of olfactory discrimination: converging evidence for common principles across phyla. Annu. Rev. Neurosci. 20, 595-631. doi: 10.1146/annurev.neuro.20.1.595

Hinterwirth, A., Zeiner, R., and Tichy, H. (2004). Olfactory receptor cells on the cockroach antennae: responses to the direction and rate of change in food odour concentration. Eur. J. Neurosci. 19, 3389-3392. doi: 10.1111/j.0953-816X.2004. 03386.x
FIGURE S3 | Cluster analysis of temporal activity patterns elicited by cineol. Based on PSTHs during the 1-s cineol stimulations, we classified 84 PN responses into four response clusters. The four response clusters are arbitrarily grouped based on the cluster dendrogram (left panel, height 30) formed by Ward's method and PSTHs. The heat map shows PSTHs with a bin of $20 \mathrm{~ms}$, and the heater color represents the higher spike activities within the bin. Red and green circles represent the responses from type1 and type2 PNs, respectively.

Husch, A., Hess, S., and Kloppenburg, P. (2008). Functional parameters of voltageactivated $\mathrm{Ca} 2+$ currents from olfactory interneurons in the antennal lobe of Periplaneta americana. J. Neurophysiol. 99, 320-332. doi: 10.1152/jn.00719.2007 Husch, A., Paehler, M., Fusca, D., Paeger, L., and Kloppenburg, P. (2009). Calcium current diversity in physiologically different local interneuron types of the antennal lobe. J. Neurosci. 29, 716-726. doi: 10.1523/jneurosci.3677-08. 2009

Igarashi, K. M., Ieki, N., An, M., Yamaguchi, Y., Nagayama, S., Kobayakawa, K., et al. (2012). Parallel mitral and tufted cell pathways route distinct odor information to different targets in the olfactory cortex. J. Neurosci. 32, 7970-7985. doi: 10.1523/JNEUROSCI.0154-12.2012

Imen, S., Christian, M., Virginie, D., and Colette, R. (2015). Intraspecific signals inducing aggregation in Periplaneta americana (Insecta: Dictyoptera). Environ. Entomol. 44, 713-723. doi: 10.1093/ee/nvv035

Ito, K., Shinomiya, K., Ito, M., Armstrong, J. D., Boyan, G., Hartenstein, V., et al. (2014). A systematic nomenclature for the insect brain. Neuron 81, 755-765. doi: 10.1016/j.neuron.2013.12.017

Kelber, C., Rössler, W., and Kleineidam, C. J. (2006). Multiple olfactory receptor neurons and their axonal projections in the antennal lobe of the honeybee Apis mellifera. J. Comp. Neurol. 496, 395-405. doi: 10.1002/cne.20930

Kirschner, S., Kleineidam, C. J., Zube, C., Rybak, J., Grunewald, B., and Rössler, W. (2006). Dual olfactory pathway in the honeybee, Apis mellifera. J. Comp. Neurol. 499, 933-952. doi: 10.1002/cne.21158

Knaden, M., and Hansson, B. S. (2014). Mapping odor valence in the brain of flies and mice. Curr. Opin. Neurobiol. 24, 34-38. doi: 10.1016/j.conb.2013.08.010

Krofczik, S., Menzel, R., and Nawrot, M. P. (2008). Rapid odor processing in the honeybee antennal lobe network. Front. Comput. Neurosci. 2:9. doi: 10.3389/ neuro.10.009.2008

Kropf, J., Bieringer, K., Kelber, C., and Rössler, W. (2014). Olfactory subsystems in the honeybee: sensory supply and sex specificity. Cell Tissue Res. 357, 583-595. doi: 10.3389/conf.fnbeh.2012.27.00239

Malun, D., Waldow, U., Kraus, D., and Boeckh, J. (1993). Connections between the deutocerebrum and the protocerebrum, and neuroanatomy of several classes of deutocerebral projection neurons in the brain of male Periplaneta americana. J. Comp. Neurol. 329, 143-162. doi: 10.1002/cne.903290202

Martin, J. P., Beyerlein, A., Dacks, A. M., Reisenman, C. E., Riffell, J. A., Lei, H., et al. (2011). The neurobiology of insect olfaction: sensory processing in a comparative context. Prog. Neurobiol. 95, 427-447. doi: 10.1016/j.pneurobio. 2011.09.007

Mizunami, M., Iwasaki, M., Okada, R., and Nishikawa, M. (1998). Topography of four classes of Kenyon cells in the mushroom bodies of the cockroach. J. Comp. Neurol. 399, 162-175. doi: 10.1002/(SICI)1096-9861(19980921)399: $2<162:$ :AID-CNE2<3.0.CO;2-Z

Muller, D., Abel, R., Brandt, R., Zockler, M., and Menzel, R. (2002). Differential parallel processing of olfactory information in the honeybee, Apis mellifera $\mathrm{L}$. J. Comp. Physiol. A 188, 359-370. doi: 10.1007/s00359-002-0310-1

Nagayama, S., Takahashi, Y. K., Yoshihara, Y., and Mori, K. (2004). Mitral and tufted cells differ in the decoding manner of odor maps in the rat olfactory bulb. J. Neurophysiol. 91, 2532-2540. doi: 10.1152/jn.01266.2003

Nishikawa, M., Watanabe, H., and Yokohari, F. (2012). Higher brain centers for social tasks in worker ants, Camponotus japonicus. J. Comp. Neurol. 520, 1584-1598. doi: 10.1002/cne.23001

Nishino, H., Iwasaki, M., Kamimura, I., and Mizunami, M. (2012a). Divergent and convergent projections to the two parallel olfactory centers from two neighboring, pheromone-receptive glomeruli in the male American cockroach. J. Comp. Neurol. 520, 3428-3445. doi: 10.1002/cne.23111

Nishino, H., Iwasaki, M., and Mizunami, M. (2011). Pheromone detection by a pheromone emitter: a small sex pheromone-specific processing system in the 
female American cockroach. Chem. Senses 36, 261-270. doi: 10.1093/chemse/ bjq122

Nishino, H., Iwasaki, M., Yasuyama, K., Hongo, H., Watanabe, H., and Mizunami, M. (2012b). Visual and olfactory input segregation in the mushroom body calyces in a basal neopteran, the American cockroach. Arthropod Struct. Dev. 41, 3-16. doi: 10.1016/j.asd.2011.08.005

Nishino, H., Nishikawa, M., Mizunami, M., and Yokohari, F. (2009). Functional and topographic segregation of glomeruli revealed by local staining of antennal sensory neurons in the honeybee Apis mellifera. J. Comp. Neurol. 515, 161-180. doi: 10.1002/cne.22064

Nishino, H., Yamashita, S., Yamazaki, Y., Nishikawa, M., Yokohari, F., and Mizunami, M. (2003). Projection neurons originating from thermo- and hygrosensory glomeruli in the antennal lobe of the cockroach. J. Comp. Neurol. 455, 40-55. doi: 10.1002/cne.10450

Papadopoulou, M., Cassenaer, S., Nowotny, T., and Laurent, G. (2011). Normalization for sparse encoding of odors by a wide-field interneuron. Science 332, 721-725. doi: 10.1126/science.1201835

Rössler, W., and Brill, M. (2013). Parallel processing in the honeybee olfactory pathway: structure, function, and evolution. J. Comp. Physiol. A 199, 981-996. doi: 10.1007/s00359-013-0821-y

Rössler, W., and Zube, C. (2011). Dual olfactory pathway in hymenoptera: evolutionary insights from comparative studies. Arthropod. Struct. Dev. 40, 349-357. doi: 10.1016/j.asd.2010.12.001

Sakura, M., Okada, R., and Mizunami, M. (2002). Olfactory discrimination of structurally similar alcohols by cockroaches. J. Comp. Physiol. A 188, 787-797. doi: 10.1007/s00359-002-0366-y

Salecker, I., and Boeckh, J. (1995). Embryonic development of the antennal lobes of a hemimetabolous insect, the cockroach Periplaneta americana: light and electron microscopic observations. J. Comp. Neurol. 352, 33-54. doi: 10.1002/ cne.903520104

Strausfeld, N. J., and Li, Y. (1999a). Organization of olfactory and multimodal afferent neurons supplying the calyx and pedunculus of the cockroach mushroom bodies. J. Comp. Neurol. 409, 603-625. doi: 10.1002/(SICI)10969861(19990712)409:4<603::AID-CNE7<3.0.CO;2-P

Strausfeld, N. J., and Li, Y. (1999b). Representation of the calyces in the medial and vertical lobes of cockroach mushroom bodies. J. Comp. Neurol. 409, 626-646. doi: 10.1002/(SICI)1096-9861(19990712)409:4<626::AID-CNE8<3.0.CO;2-B

Takahashi, N., Katoh, K., Watanabe, H., Nakayama, Y., Iwasaki, M., Mizunami, M., et al. (2017). Complete identification of four giant interneurons supplying mushroom body calyces in an insect brain. J. Comp. Neurol. 525, 204-230. doi: $10.1002 /$ cne. 24108

Tirindelli, R., Dibattista, M., Pifferi, S., and Menini, A. (2009). From pheromones to behavior. Physiol. Rev. 89, 921-956. doi: 10.1152/physrev.00037.2008
Watanabe, H., Ai, H., and Yokohari, F. (2012a). Spatio-temporal activity patterns of odor-induced synchronized potentials revealed by voltage-sensitive dye imaging and intracellular recording in the antennal lobe of the cockroach. Front. Syst. Neurosci. 6:55. doi: 10.3389/fnsys.2012.00055

Watanabe, H., Haupt, S. S., Nishino, H., Nishikawa, M., and Yokohari, F. (2012b). Sensillum-specific, topographic projection patterns of olfactory receptor neurons in the antennal lobe of the cockroach Periplaneta americana. J. Comp. Neurol. 520, 1687-1701. doi: 10.1002/cne.23007

Watanabe, H., Kobayashi, Y., Sakura, M., Matsumoto, Y., and Mizunami, M. (2003). Classical olfactory conditioning in the cockroach Periplaneta americana. Zool. Sci. 20, 1447-1454. doi: 10.2108/zsj.20.1447

Watanabe, H., Matsumoto, C. S., Nishino, H., and Mizunami, M. (2011). Critical roles of mecamylamine-sensitive mushroom body neurons in insect olfactory learning. Neurobiol. Learn. Mem. 95, 1-13. doi: 10.1016/j.nlm.2010.10.004

Watanabe, H., Nishino, H., Nishikawa, M., Mizunami, M., and Yokohari, F. (2010). Complete mapping of glomeruli based on sensory nerve branching pattern in the primary olfactory center of the cockroach Periplaneta americana. J. Comp. Neurol. 518, 3907-3930. doi: 10.1002/cne.22452

Wilson, R. I., Turner, G. C., and Laurent, G. (2004). Transformation of olfactory representations in the Drosophila antennal lobe. Science 303, 366-370. doi: 10.1126/science.1090782

Yamagata, N., Schmuker, M., Szyszka, P., Mizunami, M., and Menzel, R. (2009). Differential odor processing in two olfactory pathways in the honeybee. Front. Syst. Neurosci. 3:16. doi: 10.3389/neuro.06.016.2009

Zube, C., Kleineidam, C. J., Kirschner, S., Neef, J., and Rösler, W. (2008). Organization of the olfactory pathway and odor processing in the antennal lobe of the ant Camponotus floridanus. J. Comp. Neurol. 506, 425-441. doi: 10.1002/cne.21548

Zwaka, H., Münch, D., Manz, G., Menzel, R., and Rybak, J. (2016). The circuitry of olfactory projection neurons in the brain of the honeybee, Apis mellifera. Front. Neuroanat. 10:90. doi: 10.3389/fnana.2016.00090

Conflict of Interest Statement: The authors declare that the research was conducted in the absence of any commercial or financial relationships that could be construed as a potential conflict of interest.

Copyright (C) 2017 Watanabe, Nishino, Mizunami and Yokohari. This is an openaccess article distributed under the terms of the Creative Commons Attribution License (CC BY). The use, distribution or reproduction in other forums is permitted, provided the original author(s) or licensor are credited and that the original publication in this journal is cited, in accordance with accepted academic practice. No use, distribution or reproduction is permitted which does not comply with these terms. 